Research Article

\title{
Earthquake and Wave Analysis of Circular Cylinder considering Water-Structure-Soil Interaction
}

\author{
Piguang Wang $(\mathbb{D}$, Yifu Chang, Mi Zhao $\mathbb{D}$, and Junyan Han \\ Key Laboratory of Urban Security and Disaster Engineering of Ministry of Education, Beijing University of Technology, \\ Beijing 100124, China \\ Correspondence should be addressed to Mi Zhao; zhaomi@bjut.edu.cn
}

Received 25 July 2019; Revised 19 June 2020; Accepted 9 July 2020; Published 28 July 2020

Academic Editor: Agathoklis Giaralis

Copyright $(92020$ Piguang Wang et al. This is an open access article distributed under the Creative Commons Attribution License, which permits unrestricted use, distribution, and reproduction in any medium, provided the original work is properly cited.

Offshore structures in zones of active seismicity are under a potential threat caused by the combined action of earthquakes and waves. Taking a submerged circular cylinder as the prototype and considering water-structure-soil interaction, the present study is devoted to the investigation of the combined action of earthquakes and waves. Water-cylinder interaction and soil-structure interaction are simulated by added mass and rigid circular massless foundations, respectively. Based on the radiation and diffraction wave theory, the scaled boundary finite element method is utilized to determine the earthquake-induced and waveinduced pressure on a circular cylinder. Then, a closed-form expression for the natural frequencies and mode shapes of the system is derived by using the transfer matrix method, where the transfer matrix is obtained based on Euler-Bernoulli's beam differential equation. Furthermore, the dynamic response of the system under the combined action of earthquakes and waves is derived by using the mode superposition method. Finally, the effects of the hydrodynamic force, wave force, and soil-structure interaction on the dynamic response of the submerged cylinder are investigated. The results indicate that the wave forces can substantially increase the dynamic responses of the cylinder and that the influence increases as the stiffness ratio increases and the width-depth ratio decreases. It is necessary to consider the combined action of earthquakes and waves in the seismic design of offshore structures.

\section{Introduction}

An increasing number of offshore structures, such as seacrossing bridges, artificial islands, and offshore wind turbines, have been constructed around the world in recent years especially in China. Ocean wave loads are the most important environmental load to consider for the design of offshore structures. However, earthquake loads will dominate the design in zones with high seismic intensities, such as the eastern coast of China and the western coast of the USA. This means that an earthquake can coincide with a wave condition [1]. Therefore, to guarantee the reliability of offshore structures, it is necessary to investigate the dynamic response of offshore structures under the combined action of earthquakes and waves. This paper takes a submerged circular cylinder as the prototype to study the earthquake and wave responses of offshore structures including waterstructure-soil interaction.
The dynamic analysis of offshore structures requires special considerations because of the fluid-structure interaction, which does not occur for onshore structures. The fluid-structure interaction produces an additional hydrodynamic force on the structure when it vibrates in water. Studies show that the additional hydrodynamic force can modify the dynamic properties of the structure and result in structural damage [2-4]. Earthquake-induced hydrodynamic forces on a circular cylinder have been studied by many researchers [5] who initially investigated the effects of hydrodynamic forces on the earthquake response of cantilever circular cylinders. The results indicated that the water compressibility is negligible for slender cylinders. The surface waves have no influence on earthquake-induced hydrodynamic forces except at low loading frequencies and will have little consequence on the seismic response of cylinders surrounded by water. The seismic responses of circular cylinders were further investigated by Williams [6] and 


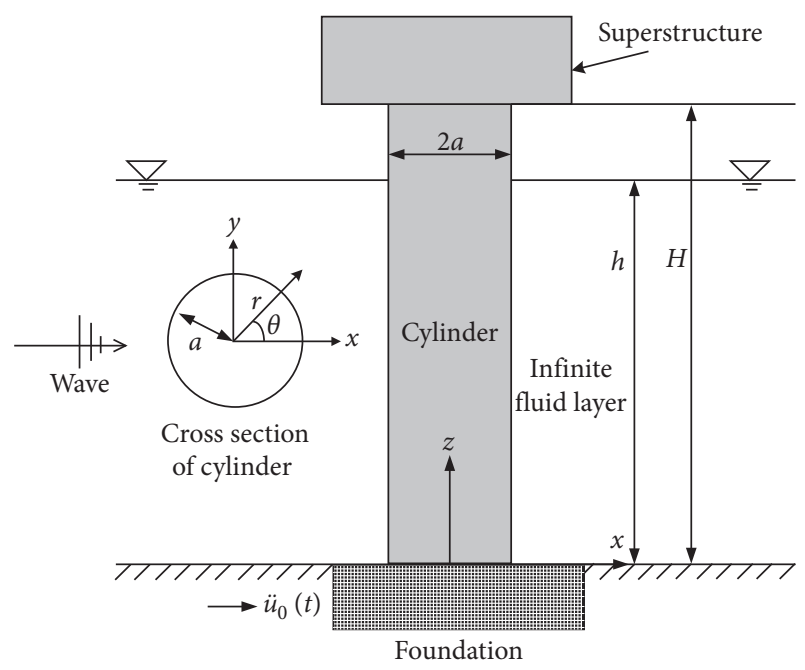

Figure 1: The fluid-structure interaction problem with rigid foundation.

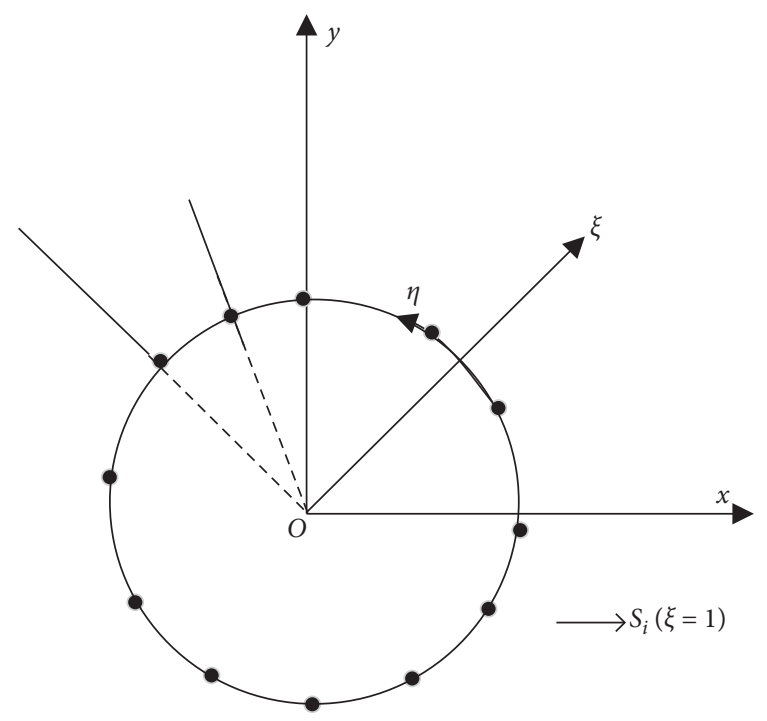

FIgURE 2: The scaled boundary coordinates.

Tanaka and Hudspeth [7]. Considering water compressibility, Du et al. [8] proposed a simplified formula for the hydrodynamic force on a circular cylinder in the time domain and Wang et al. [9] proposed an accurate time-domain model to replace the 3D infinite water layer in the watercylinder interaction analysis. The effects of the surrounding water can be entirely equivalent to an added mass when water compressibility is neglected. The simplified methods for evaluating the added hydrodynamic mass for circular, elliptical, round-ended, and rectangular cylinders were given by Goyal and Chopra [10]; Li and Yang [11]; Yang and Li [12]; Jiang et al. [13]; and Wang et al. [14-16].

Wave forces acting on vertical cylinders have also been investigated by many researchers. When the wavelength is substantially longer than the cylinder diameter, the Morison equation [17] can be used to estimate the wave forces on slender cylinders. The Morison equation is made up of two components, including a viscous drag force and an inertia force. However, for large offshore structures, the viscous drag force can be neglected. MacCamy and Fuchs [18] developed the diffraction wave theory to evaluate the wave forces on large circular cylinders immersed in water. Furthermore, the analytical solutions to calculate the wave forces on elliptical cylinders and cylinders with an arbitrary smooth cross section were proposed by Chen and Mei [19]; Williams [20]; and Liu et al. [21]. Li et al. [22] developed a semianalytical solution method to calculate the wave forces on cylinders with arbitrary shapes.

Nevertheless, only a few studies have been carried out to investigate the dynamic response of offshore structures subjected to combined earthquake and wave-current action. Penzien et al. [23] studied the dynamic responses of fixedbase offshore towers subjected to random waves and earthquakes. Liu et al. [24] conducted an experiment to study the combined action of earthquakes, waves, and currents on a pile group cable-stayed bridge tower foundation. Ding et al. [25] performed underwater shaking table tests to investigate the effect of wave-current action on the seismic responses of piers. It should be noted that the soilstructure interaction was not taken into account. Studies have indicated that the soil-structure interaction may have a substantial influence on the dynamic response of offshore structures [26]. Considering the water-structure-soil interaction, Goyal and Chopra [10,27] and Xu and Spyrakos [28] investigated the water-structure interaction and soil-structure interaction on the seismic responses of intake towers. The results showed that the seismic response was increased by the water-structure interaction and decreased by the soilstructure interaction. Yamada et al. [29] studied the dynamic response of offshore towers considering soil-structure interaction subjected to random waves and random earthquake ground motions. It was observed that the effects of wave forces on the seismic response became important with increasing tower period or water depth. This paper presents a simple model to compute the dynamic responses of circular cylinders including water-structure-soil interaction under the combined action of earthquakes and waves. The effects of water-structure interaction and soil-structure interaction are systematically analyzed.

\section{Mathematical Formulation}

The water-structure-soil interaction problem in three-dimensional space is shown in Figure 1. A circular cylinder with radius $a$ and height $H$ is situated in water with a mean depth $h$. The mass of the superstructure is $M_{S}$. The soilstructure interaction is considered to be a rigid circular massless foundation with radius $R_{0}$ on deep soil strata, where the translational and rotational stiffness of the foundation are denoted by spring constants $k_{h}$ and $k_{r}$, respectively. The foundation has an acceleration time history of the earthquake motion $\ddot{u}_{g}(t)$ along the $x$-direction. The Cartesian coordinate system $(x, y, z)$ is defined with an origin located on the seabed level with the $z$-axis directed vertically upwards. The center of the cylinder is taken as the origin of the polar coordinate system $(r, \theta)$, where $\theta$ is measured 


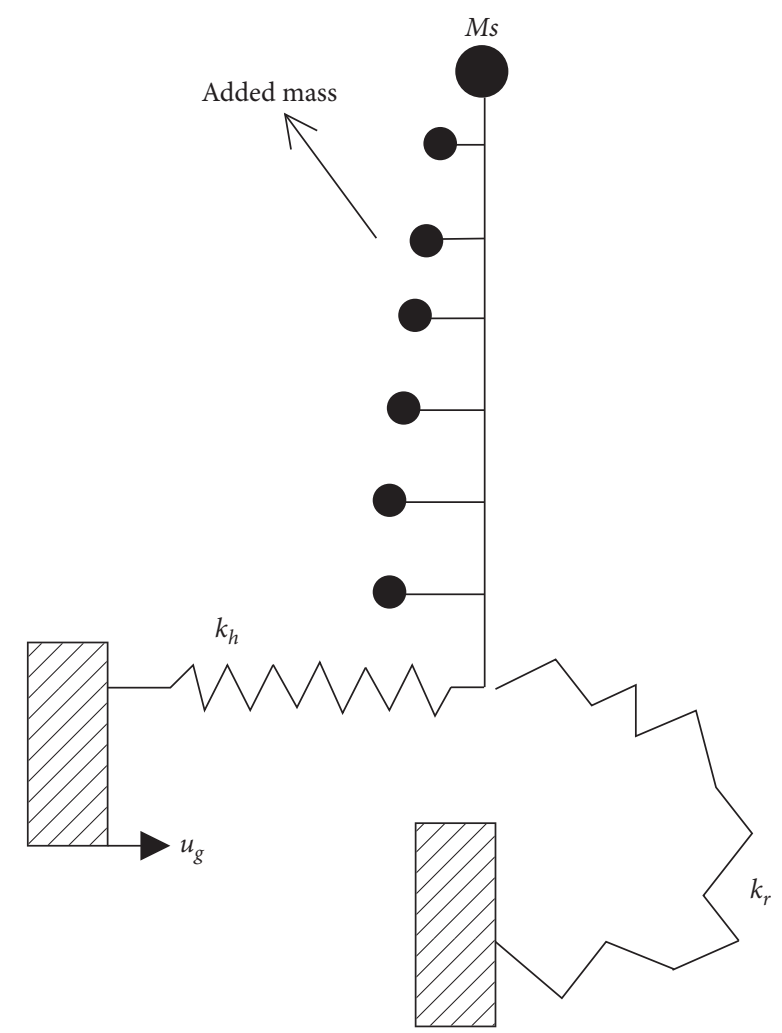

Figure 3: Simplified model of the fluid-cylinder-soil system.

counterclockwise from the positive $x$-axis. The fluid is assumed to be incompressible and inviscid. The system is initially at rest.

The Euler-Bernoulli beam theory is adopted for the dynamic analysis of the cylinder. The differential equations of the cylinder subjected to wave and earthquake actions can be expressed as follows:

$$
E I_{c} \frac{\partial^{4} u}{\partial z^{4}}+\rho_{c} A_{c} \ddot{u}=-\rho_{c} A_{c} \ddot{u}_{g}+f_{e}(z)+f_{w}(z),
$$

where $u$ and $\ddot{u}$ are the transverse deflection and acceleration along the $z$-direction, $E I_{c}$ is the bending stiffness of the pile, $\rho_{c}$ is the density of the cylinder, $A_{c}$ is the cross-sectional area of the cylinder, $f_{e}$ is the earthquake-induced hydrodynamic force on the cylinder, and $f_{w}$ is the wave force on the cylinder.

In the polar coordinate system, the earthquake-induced hydrodynamic force can be expressed as follows [16]:

$$
f_{e}=-\rho_{w}\left(\ddot{u}_{g}+\ddot{u}\right)\left[\int_{0}^{2 \pi} \sum_{j=1}^{\infty} P_{j}(r, \theta) a \cos \theta d \theta \cos \lambda_{j} z\right],
$$

where $\lambda_{j}=(2 j-1) \pi / 2 h$ and the hydrodynamic pressure $P_{j}(r, \theta, t)$ is governed by a two-dimensional Helmholtz equation with the boundary conditions on the surface of the cylinder as follows:

$$
\begin{aligned}
\nabla^{2} P_{j}-\lambda_{j}^{2} P_{j} & =0, \\
\left.\frac{\partial P_{j}}{\partial r}\right|_{r=a} & =-\frac{2(-1)^{j+1}}{\lambda_{j} h} \cos \theta,
\end{aligned}
$$

in which $\rho_{w}$ is the water density and $\nabla^{2}=\left(\partial^{2} / \partial r^{2}\right)+$ $(1 / r)(\partial / \partial r)+\left(1 / r^{2}\right)\left(\partial^{2} / \partial \theta^{2}\right)$ is the Laplace operator.

Assuming that the wavelength is substantially longer than the cylinder diameter, the wave force can be expressed as [21]

$$
\begin{aligned}
& f_{w}=-\frac{\rho g H_{w}}{2} \frac{\cos h k z}{\cos h k h} \operatorname{Re}\left[\int_{0}^{2 \pi}\left[P_{I}+P_{S}\right] e^{-i \omega_{0} t} a \cos \theta \mathrm{d} \theta\right], \\
& P_{I}=-e^{i k r \cos \theta}
\end{aligned}
$$

where $i=\sqrt{-1}, g$ is the gravitational acceleration, $H_{w}$ is the wave height, $k$ is the wavenumber, $\omega_{0}=\sqrt{g k \tan h k h}$ is the wave frequency, $P_{I}$ is the incident wave pressure, and $P_{S}$ is the scattered wave pressure. In the polar coordinate system, $P_{S}$ is governed by a two-dimensional Helmholtz equation with the boundary conditions on the surface of the cylinder as follows:

$$
\begin{gathered}
\nabla^{2} P_{S}+k^{2} P_{S}=0 \\
\left.\frac{\partial P_{S}}{\partial r}\right|_{r=a}=-\left.\frac{\partial P_{I}}{\partial r}\right|_{r=a}=i k \cos \theta e^{i k a \cos \theta} .
\end{gathered}
$$

In addition, the radiation pressure waves $P_{j}$ and $P_{S}$ propagate away from the structure and decay with the traveling distance. The range of the applications of the present wave model is $2 a / L_{0}>0.2$, where $L_{0}$ represents the wavelength.

\section{Earthquake-Induced and Wave- Induced Pressures}

The scaled boundary finite element method (SBFEM) developed by Wolf [30] was used to determine the earthquake-induced and wave-induced pressures on a circular cylinder. The formulation of the scaled boundary finite element (SBFE) governing equations of the $2 \mathrm{D}$ pressure $P_{j}$ or $P_{s}$ is derived by converting (3) or (6) into the scaled boundary coordinates with the scaling center at the center of the cylinder in the present study, as shown in Figure 2. The circumferential coordinate $\eta$ is the anticlockwise direction along the defining curve $S$, which is closed in this case. The normalized radial coordinate $\xi$ defined as 1 at curve $S$ is a scaling factor with $1 \leq \xi \leq \infty$ for the infinite water domain. Therefore, the polar coordinates are transformed to the scaled boundary coordinates $\xi$ and $\eta$ with the following scaling equations: 


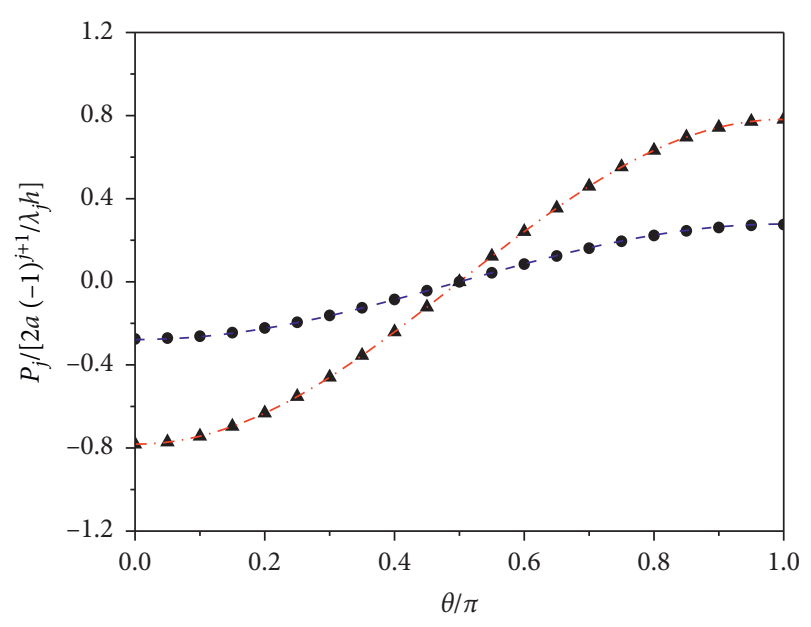

$$
\begin{aligned}
& \triangle \quad \lambda_{j} a=0.5 \text { FEM (Wang et al., 2019c) } \\
&-\quad \lambda_{j} a=0.5 \text { SBFEM } \\
& \text { - } \quad \lambda_{j} a=3.0 \text { FEM (Wang et al., 2019c) } \\
&--\lambda_{j} a=3.0 \text { SBFEM }
\end{aligned}
$$

Figure 4: Hydrodynamic pressures $P_{j}$ on a circular cylinder for the FEM [33] versus SBFEM.

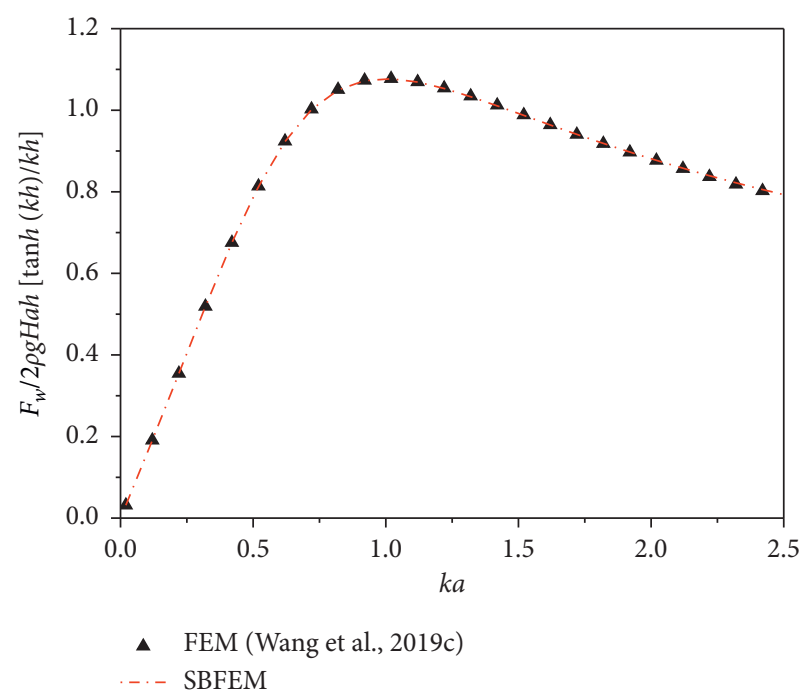

FIgURE 5: The total wave force on a circular cylinder for the FEM [33] versus SBFEM.

TABLE 1: The first natural frequency of a circular cylinder with $E=29.4 \mathrm{GPa}, \rho_{s}=2450 \mathrm{~kg} / \mathrm{m}^{3}$, and $h=20 \mathrm{~m}$.

\begin{tabular}{lccc}
\hline$a / h$ & $\begin{array}{c}\text { Reference [15] } \\
(\mathrm{rad} / \mathrm{s})\end{array}$ & $\begin{array}{c}\text { Present study } \\
(\mathrm{rad} / \mathrm{s})\end{array}$ & $\begin{array}{c}\text { Difference } \\
(\%)\end{array}$ \\
\hline 0.025 & 6.530 & 6.507 & 0.35 \\
0.030 & 7.855 & 7.827 & 0.36 \\
0.040 & 10.523 & 10.482 & 0.39 \\
0.050 & 13.212 & 13.157 & 0.42 \\
\hline
\end{tabular}

$$
\begin{aligned}
& x_{S}(\eta)=\xi r \cos \left(\frac{\eta}{r}\right), \\
& y_{S}(\eta)=\xi r \sin \left(\frac{\eta}{r}\right) .
\end{aligned}
$$

Following the concept of an isoparametric element, the pressure $P=P_{j}$ or $P=P_{s}$ at a point $(\xi, \eta)$ is calculated by

$$
P(\xi, \eta)=\mathbf{N}(\eta) \mathbf{P}(\xi),
$$

where $\mathbf{N}(\eta)$ is the shape function, the vector $\mathbf{P}(\xi)$ is the hydrodynamic pressure along the radial lines and is analytical with respect to $\xi$.

By performing a scaled boundary transformation, the operator $\nabla$ can be written as

$$
\begin{aligned}
\nabla & =\mathbf{b}_{1}(\eta) \frac{\partial}{\partial \xi}+\frac{1}{\xi} \mathbf{b}_{2}(\eta) \frac{\partial}{\partial \eta}, \\
\mathbf{b}_{1}(\eta) & =\frac{1}{|J|}\left\{\begin{array}{c}
y_{S}(\eta)_{, \eta} \\
-x_{S}(\eta)_{, \eta}
\end{array}\right\}, \\
\mathbf{b}_{2}(\eta) & =\frac{1}{|J|}\left\{\begin{array}{c}
-y_{S}(\eta) \\
x_{S}(\eta)
\end{array}\right\},
\end{aligned}
$$

where $|J|$ is related to the determinant of the Jacobian matrix at the boundary:

$$
|J|=x_{S}(\eta) y_{S}(\eta)_{, \eta}-y_{S}(\eta) x_{S}(\eta)_{, \eta}
$$

The infinitesimal volume $d V$ for any radial coordinate $\xi$ is calculated as

$$
\mathrm{d} V=|J| \xi \mathrm{d} \xi \mathrm{d} \eta
$$

The finite element method requires the weighted residuals of the governing equation in (3) or (6) to be zero. Applying the Galerkin approach, the weighting function $w$ can be chosen as the same shape function as in (9):

$$
w(\xi, \eta)=\mathbf{N}(\eta) \mathbf{w}(\xi) .
$$

3.1. Solution of the Earthquake-Induced Pressure. By applying the weighted residual method and performing integration by parts, the solution of the differential in (3) with the boundary condition in (4) is equivalent to the following weak form:

$$
\int_{V} \nabla^{T} w \nabla P_{j} \mathrm{~d} V+\lambda_{j}^{2} \int_{V} w P_{j} \mathrm{~d} V+\int_{S} w v_{j n} \mathrm{~d} S=0,
$$

where $v_{j n}=\left(2 \sin (-1)^{j+1} / \lambda_{j} h\right) \cos \theta$.

Substituting (9), (10), (13), and (14) into (15), after some manipulations, the scaled boundary finite element equation and the boundary condition equation for the earthquakeinduced $2 \mathrm{D}$ pressure can be unified as

$$
\begin{gathered}
\mathbf{E}_{0} \xi^{2} \mathbf{P}_{j}(\xi)_{, \xi \xi}+\left(\mathbf{E}_{0}-\mathbf{E}_{1}+\mathbf{E}_{1}^{T}\right) \xi \mathbf{P}_{j}(\xi)_{, \xi}-\mathbf{E}_{2} \mathbf{P}_{j}(\xi)-\lambda_{j}^{2} \xi^{2} \mathbf{M}_{0} \mathbf{P}_{j}(\xi)=0, \\
\mathbf{E}_{0} \xi \mathbf{P}_{j}(\xi)_{, \xi}+\mathbf{E}_{1}^{T} \mathbf{P}_{j}(\xi)+\mathbf{q}_{j}(\xi)=0,
\end{gathered}
$$




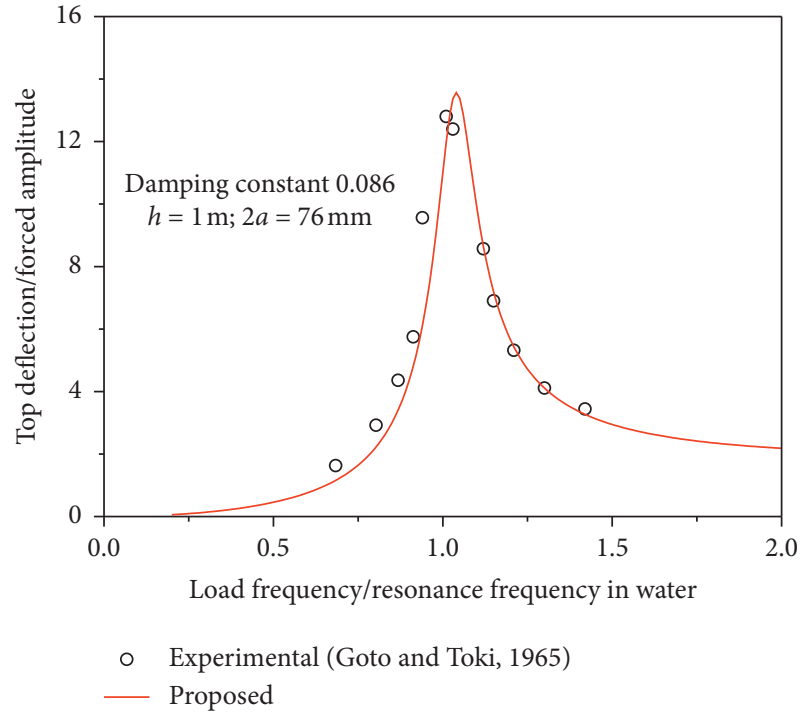

FIGURE 6: Resonance curve of a circular cylinder excited by harmonic ground motions.

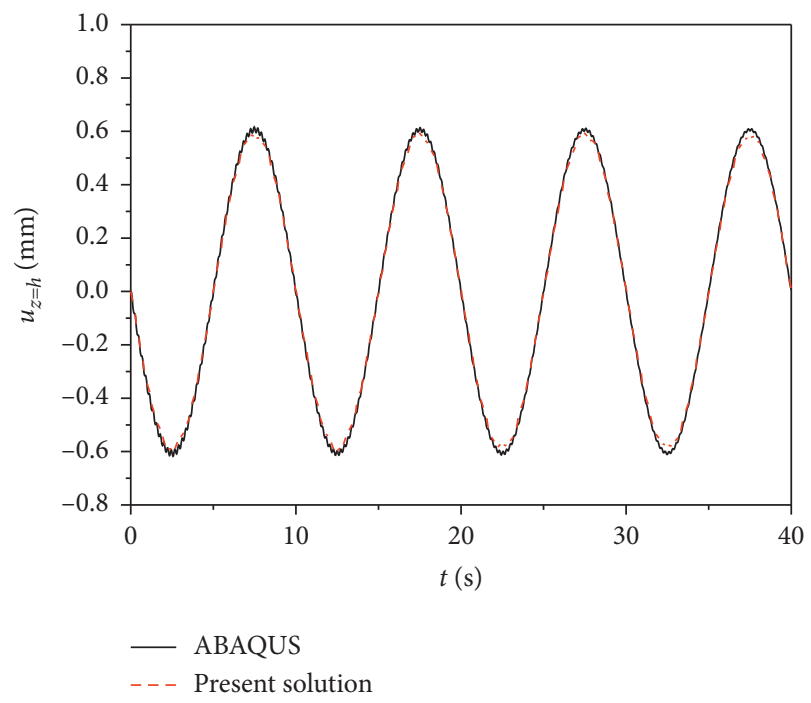

Figure 7: The time history of the displacement at $z=h$ excited by wave forces.

where $\mathbf{E}_{0}, \mathbf{E}_{1}, \mathbf{E}_{2}, \mathbf{M}_{0}$, and $\mathbf{q}_{j}$ are expressed as

$$
\begin{aligned}
\mathbf{E}_{d} & =\int_{S} \mathbf{B}_{1}(\eta)^{T} \mathbf{B}_{1}(\eta)|J| d \eta=\frac{1}{a} \int_{S} \mathbf{N}(\eta)^{T} \mathbf{N}(\eta) d \eta \\
\mathbf{E}_{1} & =\int_{S} \mathbf{B}_{2}(\eta)^{T} \mathbf{B}_{1}(\eta)|J| d \eta=0 \cdot \mathbf{I}, \\
\mathbf{E}_{2} & =\int_{S} \mathbf{B}_{2}(\eta)^{T} \mathbf{B}_{2}(\eta)|J| d \eta, \\
\mathbf{M}_{d} & =\int_{S} \mathbf{N}(\eta)^{T} \mathbf{N}(\eta)|J| d \eta=a^{2} \mathbf{E}_{d} \mathbf{I}, \\
\mathbf{q}_{j} & =\int_{S} \mathbf{N}(\eta)^{T} v_{j n}(\xi, \eta) d \eta,
\end{aligned}
$$

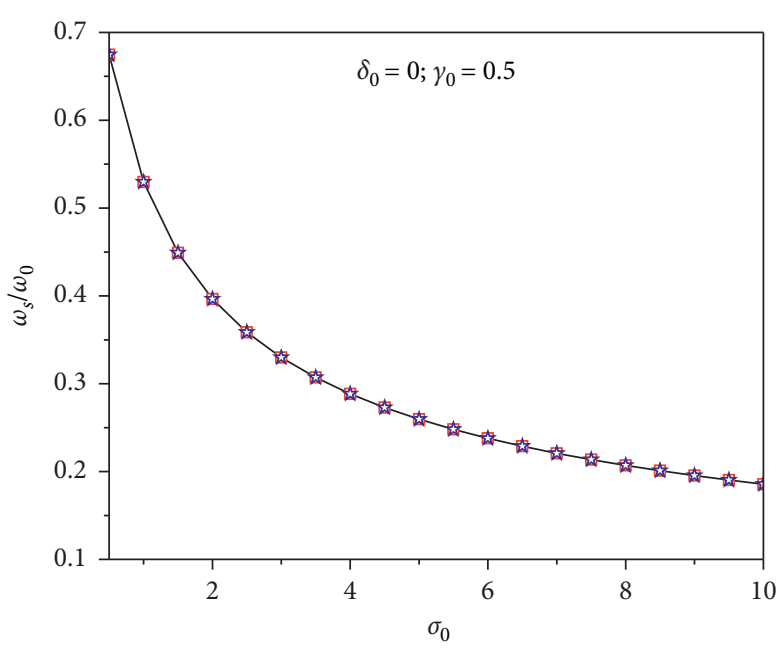

$$
\begin{aligned}
& \longrightarrow l_{0}=0.2 ; H=20 \\
& \square l_{0}=0.5 ; H=20 \\
& \text { Ł } \quad l_{0}=0.2 ; H=80
\end{aligned}
$$

FIgURE 8: The variation in the ratio $\omega_{s} / \omega_{0}$ with respect to $\sigma_{0}$ with $\delta_{0}=0$ and $\gamma_{0}=0.5$.

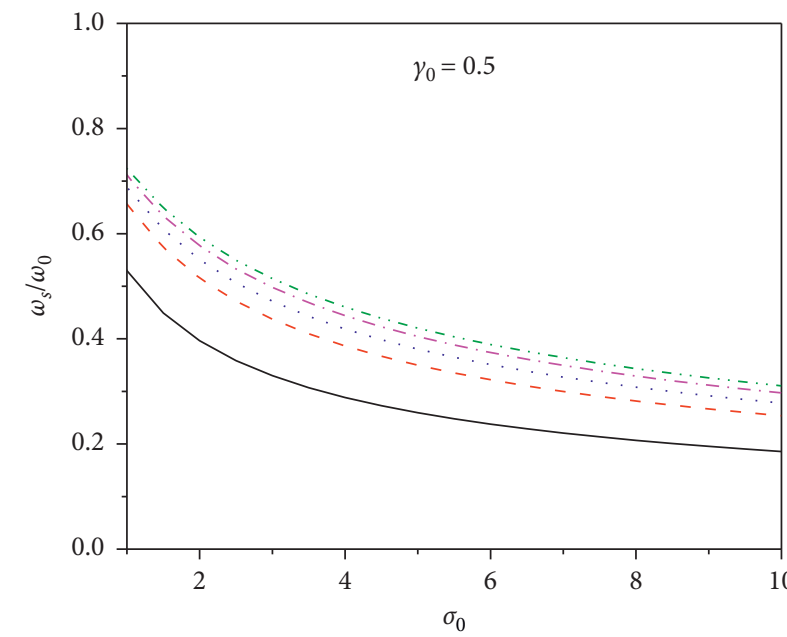

$$
\begin{aligned}
& -\delta_{0}=0 \quad \cdots \delta_{0}=2 \\
& \text { - - } \delta_{0}=0.5 \quad \ldots \delta_{0}=4 \\
& \text { ….. } \delta_{0}=1
\end{aligned}
$$

FIgURE 9: The variation in the ratio $\omega_{s} / \omega_{0}$ with respect to $\sigma_{0}$ for different values of $\delta_{0}$ with $\gamma_{0}=0.5$.

in which $\mathbf{B}_{1}(\eta)=\mathbf{b}_{1}(\eta) \mathbf{N}(\eta), \mathbf{B}_{2}(\eta)=\mathbf{b}_{2}(\eta) \mathbf{N}(\eta)_{, \eta}, \mathbf{I}$ is the identity matrix of rank $n$, and $n$ denotes the number of nodes in curve $S$.

Using $\mathbf{E}_{1}=0 \cdot \mathbf{I}$ and $\mathbf{M}_{0}=a^{2} \mathbf{E}_{0} \mathbf{I}$, premultiplying both sides of (17) by $\mathbf{E}_{0}^{-1}$ and simplifying, we get

$$
\zeta^{2} \mathbf{P}_{j}(\zeta)_{, \zeta \zeta}+\zeta \mathbf{P}_{j}(\zeta)_{, \zeta}-\mathbf{E}_{0}^{-1} \mathbf{E}_{2} \mathbf{P}_{j}(\zeta)-\zeta^{2} \mathbf{P}_{j}(\zeta)=0,
$$

where $\zeta=\lambda_{j} a \xi$. Equation (19) is the matrix form of the modified Bessel differential equation. Considering the radiation condition, it is logical to select $K_{r_{j}}(\zeta) \mathbf{T}_{j}$ as a base 


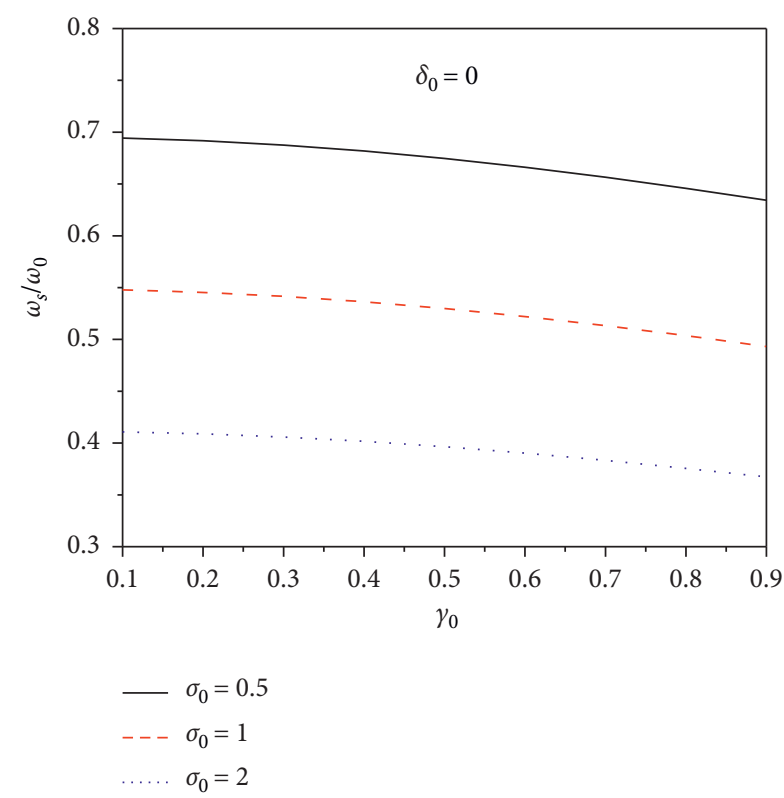

Figure 10: The variation in the ratio $\omega_{s} / \omega_{0}$ with respect to $\gamma_{0}$ for different values of $\sigma_{0}$ with $\delta_{0}=0$.

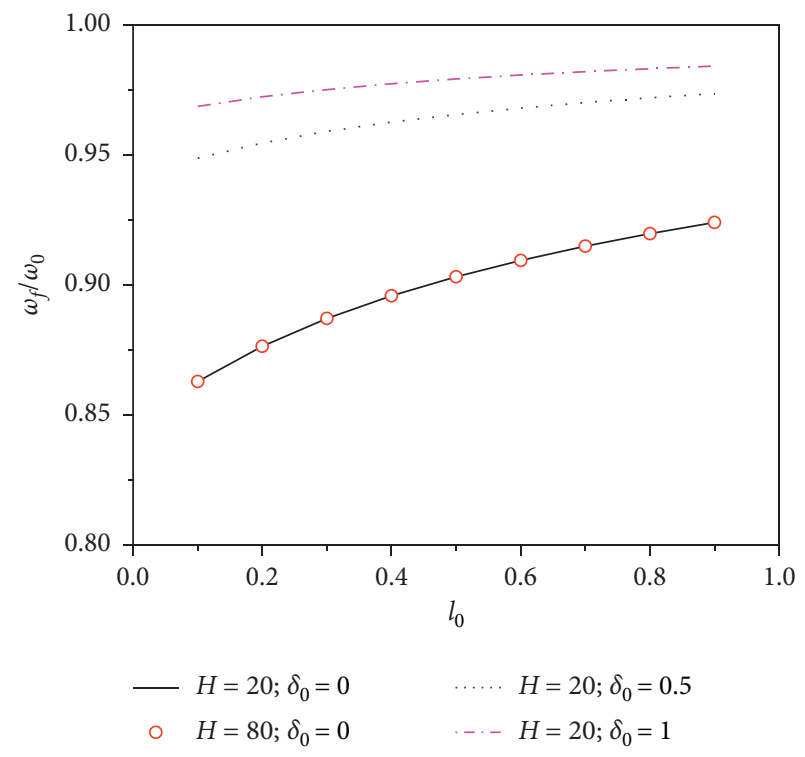

FIgURE 11: The variation in the ratio $\omega_{f} / \omega_{0}$ with respect to $l_{0}$ for different values of $\delta_{0}$ and $\mathrm{H}$.

solution for (19). Therefore, the solution for $\mathbf{P}_{j}(\zeta)$ can be expressed in the form of a series as

$$
\mathbf{P}_{j}(\zeta)=\sum_{n=1}^{N} c_{n} K_{r_{n}}(\zeta) \mathbf{T}_{n},
$$

where $c_{j}$ are coefficients, $\mathbf{T}_{j}$ are vectors of rank $n$, and $K_{r_{j}}(\cdot)$ are the modified Bessel functions of the second kind.

Substituting (20) into (19) and using the following properties of the modified Bessel function of the second kind give

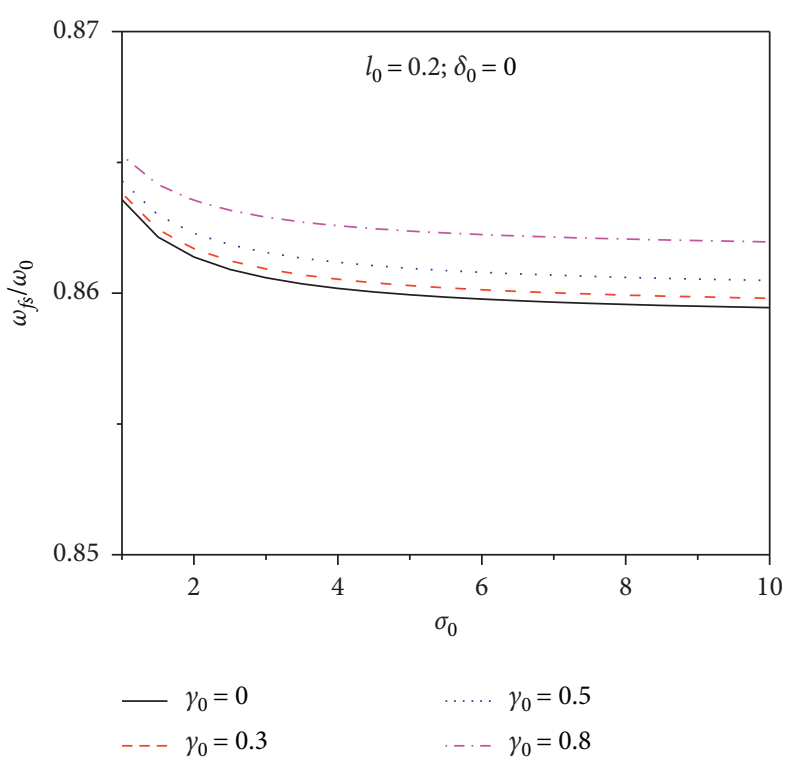

FIgURE 12: The variation in the ratio $\omega_{f s} / \omega_{s}$ with respect to $\sigma_{0}$ for different values of $\gamma_{0}$ with $\delta_{0}=0$ and $l_{0}=0.2$.

$$
\begin{aligned}
\zeta^{2} K_{r_{n}}^{\prime \prime}(\zeta) & =\zeta^{2} K_{r_{n}}(\zeta)+\zeta K_{r_{n}+1}(\zeta)-r_{n} K_{r_{n}}(\zeta)+r_{n}^{2} K_{r_{n}}(\zeta), \\
\zeta K_{r_{n}}^{\prime}(\zeta) & =-\zeta K_{r_{n}+1}(\zeta)+r_{n} K_{r_{n}}(\zeta),
\end{aligned}
$$

where the prime and the double prime represent the first and second derivatives with respect to argument $\zeta$, respectively, and we obtain

$$
\sum_{n=1}^{N}\left(\mathbf{E}_{0}^{-1} \mathbf{E}_{2}-r_{n}^{2} \mathbf{I}\right) \mathbf{T}_{n} \cdot c_{n} K_{r_{n}}(\zeta)=0
$$

Usually, $c_{n} K_{r_{n}}(\zeta) \neq 0$, so that

$$
\left(\mathbf{E}_{0}^{-1} \mathbf{E}_{2}-r_{n}^{2} \mathbf{I}\right) \mathbf{T}_{n}=0 .
$$

Let the variable $v_{n}$ be the eigenvalues of $\mathbf{E}_{0}^{-1} \mathbf{E}_{2}$; then $r_{n}=\sqrt{v_{n}}$; and $\mathbf{T}_{n}$ are the eigenvectors of $\mathbf{E}_{0}^{-1} \mathbf{E}_{2}$.

Substituting (20) into (17), we obtain

$$
\mathbf{E}_{0} \lambda_{j} a \sum_{n=1}^{N} c_{n} K_{r_{n}}^{\prime}\left(\lambda_{j} a\right) \mathbf{T}_{n}=-\mathbf{q}_{j} .
$$
as

Using (20) and (24), the pressure $\mathbf{P}_{j}(\zeta)$ can be obtained

$$
\mathbf{P}_{j}(\zeta)=-\mathbf{S}_{j}^{-1} \mathbf{q}_{j} .
$$

Here, the dynamic-stiffness matrix ${ }_{\mathbf{S}}^{j}$ is expressed as

$$
\begin{aligned}
\mathbf{S}_{j} & =\mathbf{E}_{0} \mathbf{T K}_{b k} \mathbf{T}^{-1}, \\
\mathbf{K}_{b k} & =\operatorname{diag}\left[\lambda_{j} a K_{r_{1}}^{\prime} \frac{\left(\lambda_{j} a\right)}{K_{r_{1}}\left(\lambda_{j} a\right)}, \ldots, \lambda_{j} a K_{r_{n}}^{\prime} \frac{\left(\lambda_{j} a\right)}{K_{r_{n}}\left(\lambda_{j} a\right)}\right], \\
\mathbf{T} & =\left[\mathbf{T}_{1}, \mathbf{T}_{2}, \ldots, \mathbf{T}_{m}\right],
\end{aligned}
$$




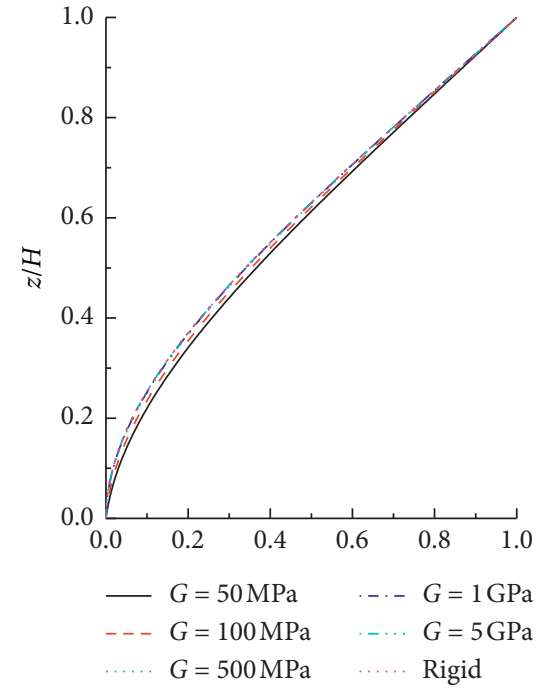

(a)

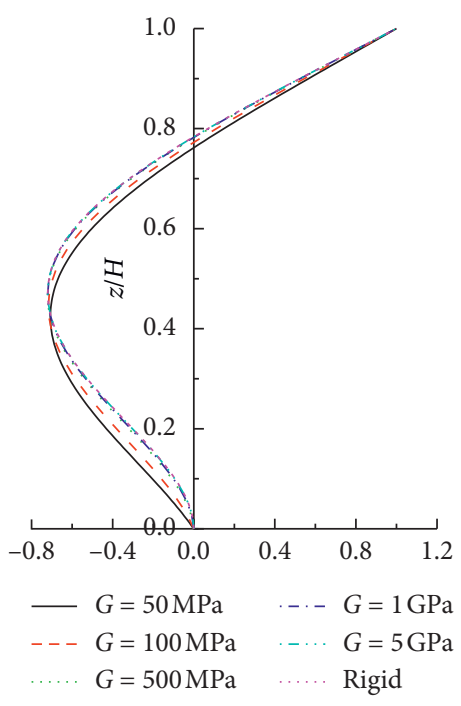

(b)

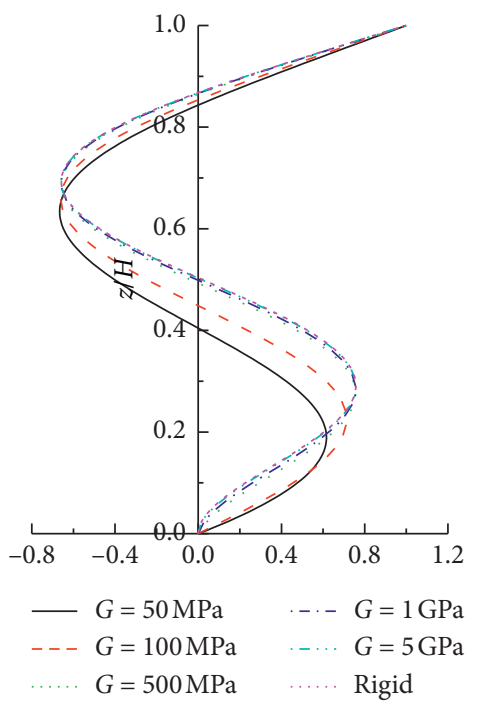

(c)

Figure 13: Vibration mode shapes in a vacuum for different values of $G$ with $H=8 \mathrm{~m}, l_{0}=0.1, \delta_{0}=0$, and $\gamma_{0}=0.5$ : (a) first, (b) second, and (c) third.

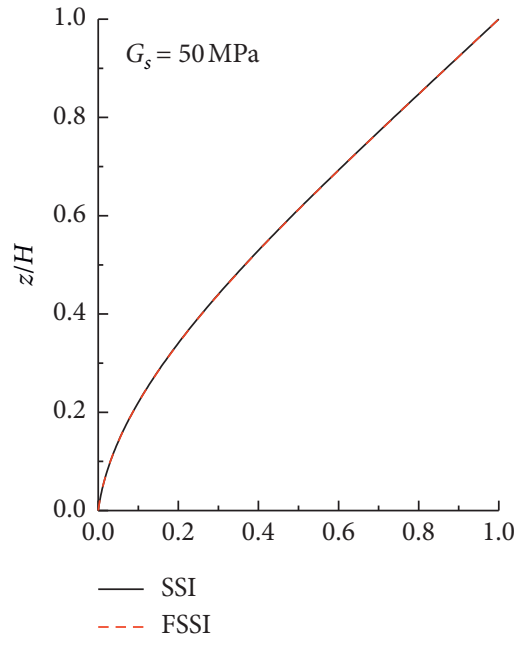

(a)

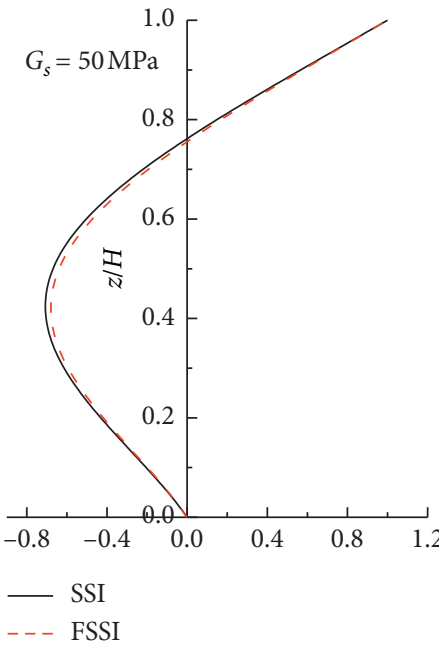

(b)

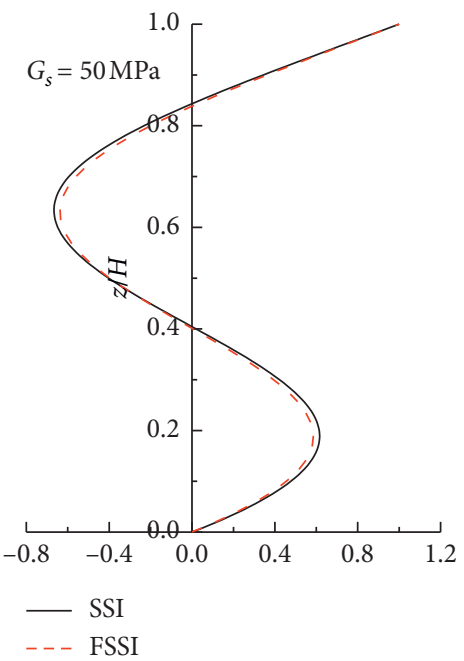

(c)

Figure 14: Vibration mode shapes in a vacuum and in water with $H=8 \mathrm{~m}, G=50 \mathrm{MPa}, l_{0}=0.1, \delta_{0}=0$, and $\gamma_{0}=0.5$ : (a) first, (b) second, and (c) third.

where "diag" represents a diagonal matrix with the elements in square brackets on the main diagonal.

3.2. Solution of the Wave-Induced Pressure. By applying the weighted residual method and performing integration by parts, the solution of the differential equation in (6) with the boundary condition in (7) is equivalent to the following weak form:

$$
\int_{V} \nabla^{T} w \nabla P_{S} \mathrm{~d} V-k^{2} \int_{V} w P_{S} \mathrm{~d} V-\int_{S} w v_{n} \mathrm{~d} S=0,
$$

where $v_{n}=\mathrm{i} k \cos \theta \mathrm{e}^{\mathrm{i} k a \cos \theta}$.
Substituting (9), (10), (13), and (14) into (27), after some manipulations, the scaled boundary finite element equation and the boundary condition equation for the wave-induced $2 \mathrm{D}$ pressure can be unified as

$$
\begin{array}{r}
\mathbf{E}_{0} \xi^{2} \mathbf{P}_{S}(\xi)_{, \xi \xi}+\left(\mathbf{E}_{0}-\mathbf{E}_{1}+\mathbf{E}_{1}^{T}\right) \xi \mathbf{P}_{S}(\xi)_{, \xi}-\mathbf{E}_{2} \mathbf{P}_{S}(\xi)+k^{2} \xi^{2} \mathbf{M}_{0} \mathbf{P}_{S}(\xi)=0, \\
\mathbf{E}_{0} \xi \mathbf{P}_{S}(\xi)_{, \xi}+\mathbf{E}_{1}^{T} \mathbf{P}_{S}(\xi)-\mathbf{q}(\xi)=0,
\end{array}
$$

where $\mathbf{q}=\int_{S} \mathbf{N}(\eta)^{T} v_{n}(\xi, \eta) 0 d$. Using $\quad \mathbf{E}_{1}=0 \cdot \mathbf{I} \quad$ and $\mathbf{M}_{0}=a^{2} \mathbf{E}_{0} \mathbf{I}$, premultiplying both sides of (28) by $\mathbf{E}_{0}^{-1}$ and simplifying, we obtain 


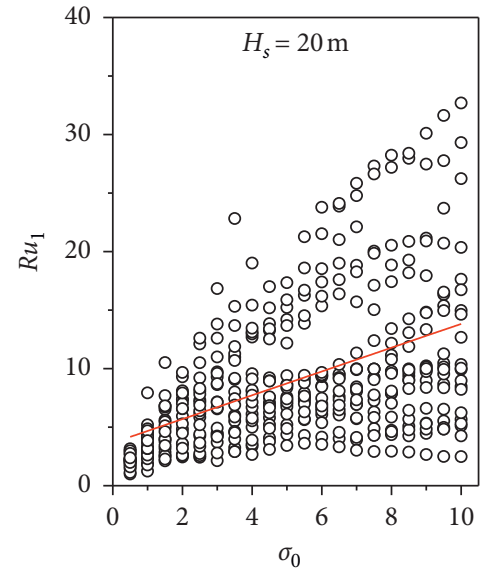

— Trend line

(a)

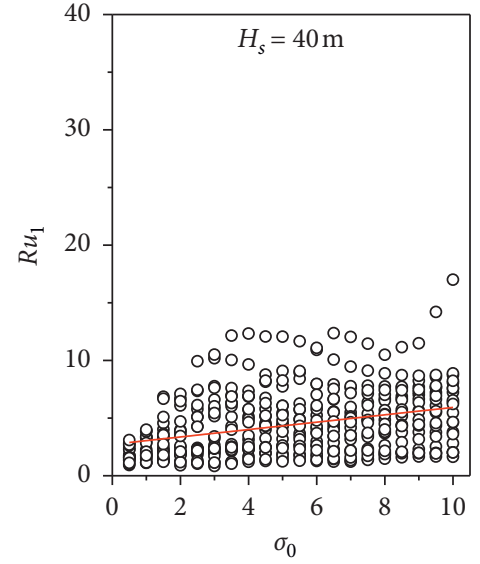

- Trend line

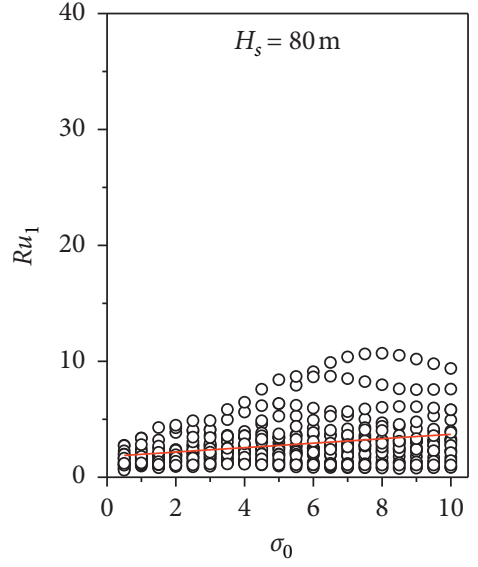

- Trend line

(b)

(c)

Figure 15: The parameters $R u_{1}$ versus $\sigma_{0}$ for different values of $\mathrm{H}$ with $l_{0}=0.2$ and $\gamma_{0}=0.5$.

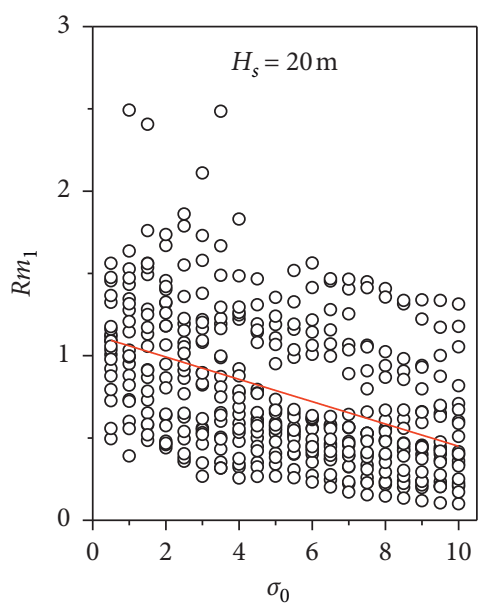

— Trend line

(a)

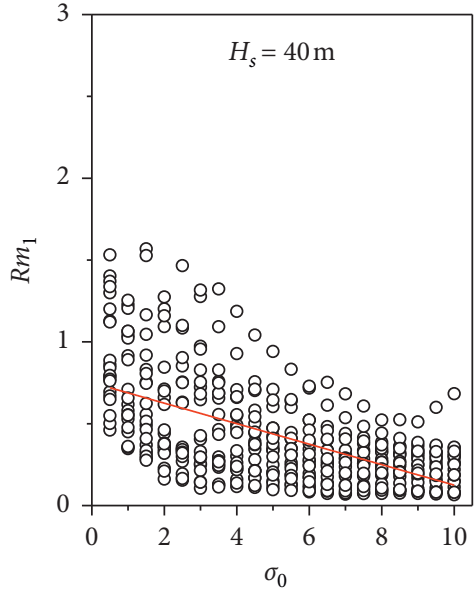

_ Trend line

(b)

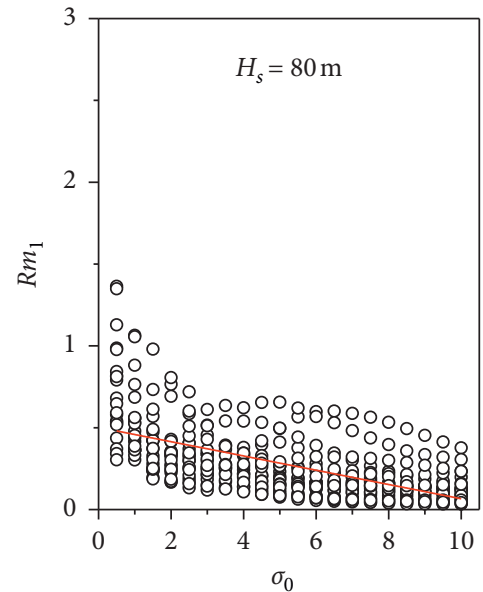

_ Trend line

(c)

FIgURE 16: The parameters $R m_{1}$ versus $\sigma_{0}$ for different values of $\mathrm{H}$ with $l_{0}=0.2$ and $\gamma_{0}=0.5$.

$$
\zeta^{2} \mathbf{P}_{S}(\zeta)_{, \zeta \zeta}+\zeta \mathbf{P}_{S}(\zeta)_{, \zeta}-\mathbf{E}_{0}^{-1} \mathbf{E}_{2} \mathbf{P}_{S}(\zeta)+\zeta^{2} \mathbf{P}_{S}(\zeta)=0
$$

where $\zeta=k a \xi$.

Equation (30) is the matrix form of Bessel's differential equation. Considering the radiation condition, it is logical to select $H_{r_{j}}(\zeta) \mathbf{T}_{j}$ as a base solution of (30). Therefore, the solution for $\mathbf{P}_{S}(\zeta)$ can be expressed in the form of a series as

$$
\mathbf{P}_{S}(\zeta)=\sum_{n=1}^{N} c_{n} H_{r_{n}}(\zeta) \mathbf{T}_{n}
$$

where $H_{r_{i}}(\cdot)$ are the Hankel functions of the first kind.

Substituting (31) into (30) and using the properties of the Hankel function give

$$
\zeta^{2} H_{r_{j}}^{\prime \prime}(\zeta)=-\zeta^{2} H_{r_{j}}(\zeta)+\zeta H_{r_{j}+1}(\zeta)-r_{j} H_{r_{j}}(\zeta)+r_{j}^{2} H_{r_{j}}(\zeta)
$$

$$
\zeta H_{r_{j}}^{\prime}(\zeta)=-\zeta H_{r_{j}+1}(\zeta)+r_{j} H_{r_{j}}(\zeta)
$$

We obtain

$$
\sum_{n=1}^{N}\left(\mathbf{E}_{0}^{-1} \mathbf{E}_{2}-r_{n}^{2} \mathbf{I}\right) \mathbf{T}_{n} \cdot c_{n} H_{r_{n}}(\zeta)=0
$$

Substituting (33) into (29), we obtain

$$
\mathbf{E}_{0} k a \sum_{n=1}^{N} c_{n} H_{r_{n}}^{\prime}(k a) \mathbf{T}_{n}=\mathbf{M}_{1} \mathbf{v}_{n} .
$$




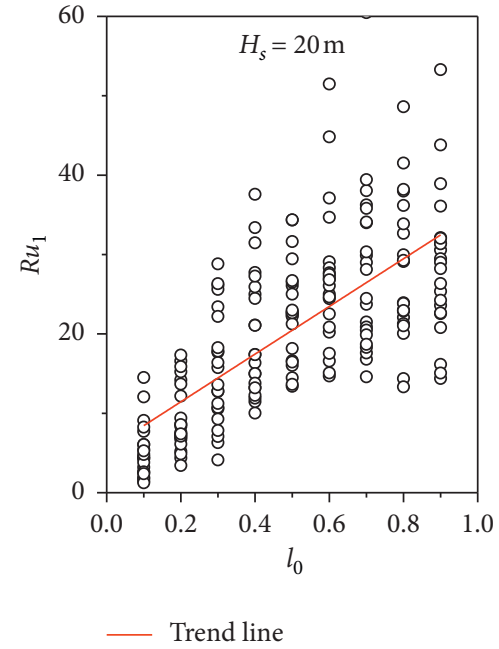

(a)

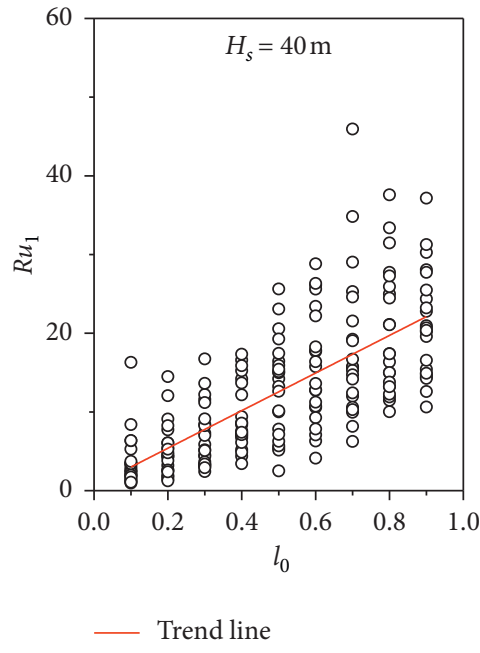

(b)

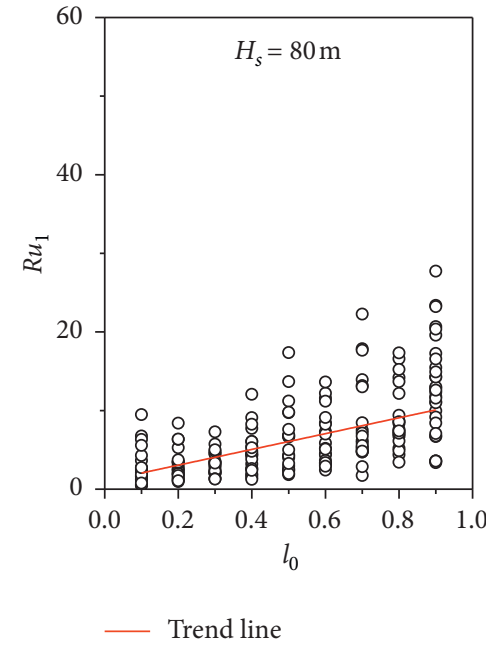

(c)

Figure 17: The parameters $R u_{1}$ versus $l_{0}$ for different values of $\mathrm{H}$ with $\sigma_{0}=5$ and $\gamma_{0}=0.5$.

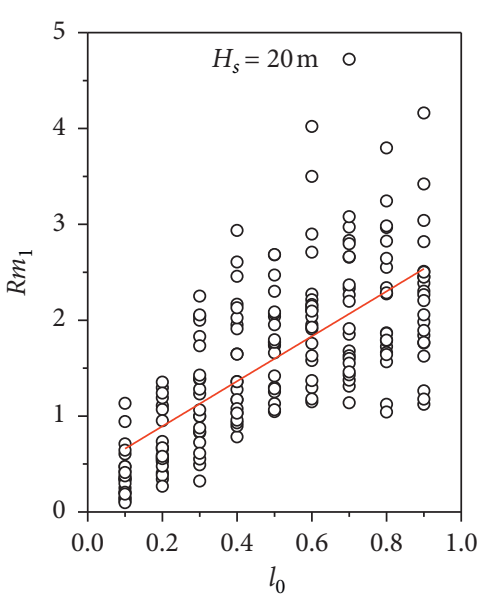

— Trend line

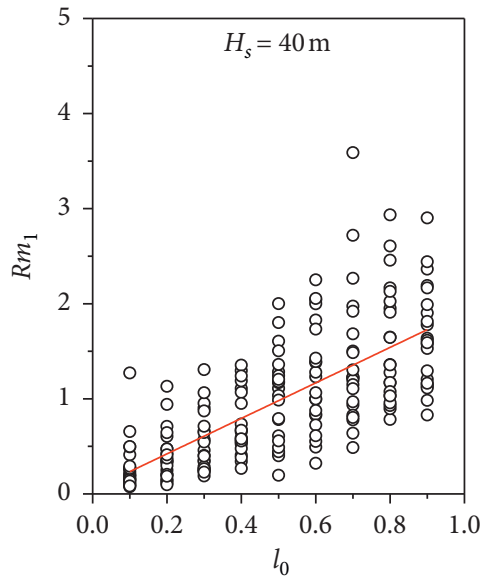

Trend line

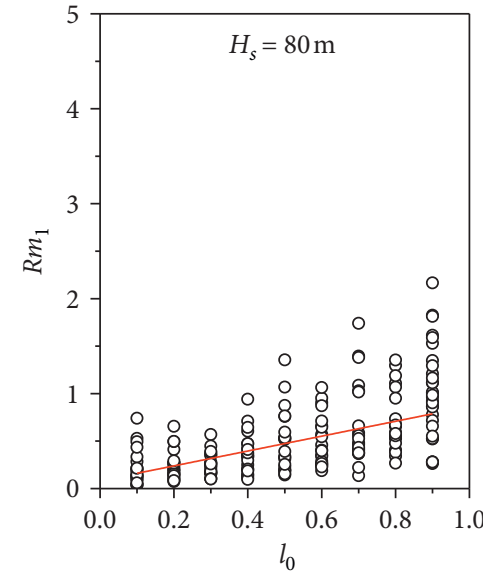

_ Trend line

(c)

(a)

(b)

Figure 18: The parameters $R m_{1}$ versus $l_{0}$ for different values of $\mathrm{H}$ with $\sigma_{0}=5$ and $\gamma_{0}=0.5$. as

Using (34) and (35), the pressure $\mathbf{P}_{S}(\zeta)$ can be obtained

$$
\mathbf{P}_{j}(\zeta)=\mathbf{S}_{w}^{-1} \mathbf{M}_{1} \mathbf{v}_{n}
$$

where the dynamic-stiffness matrix $\underset{\mathbf{S}}{w}$ is expressed as

$$
\begin{array}{r}
\mathbf{S}_{w}=\mathbf{E}_{0} \mathbf{T} \mathbf{H}_{b h} \mathbf{T}^{-1}, \\
\mathbf{H}_{b h}=\operatorname{diag}\left[k a H_{r_{1}}^{\prime} \frac{(k a)}{H_{r_{1}}(k a)}, \ldots, k a H_{r_{n}}^{\prime} \frac{(k a)}{H_{r_{n}}(k a)}\right] .
\end{array}
$$

\section{Solution of the Equations of Motion}

The simplified model of the fluid-cylinder-soil system is shown in Figure 3. The mode superposition method is utilized to solve the equations of motion in the present study. The equations of motion of the free vibration are first solved, and the results are further used to solve the dynamic responses of the cylinder under the combined action of waves and earthquakes.

The free vibration equation of the cylinder vibrating in water can be written as

$$
E I_{c} \frac{\partial^{4} u}{\partial z^{4}}+\rho_{c} A_{c} \ddot{u}=-m_{w}(z) \ddot{u},
$$

where $m_{w}(z)=\rho_{w} \sum_{j=1}^{\infty} \int_{0}^{2 \pi} P_{j} a \cos \theta \mathrm{d} \theta \cos \lambda_{j} z$ denotes the added mass on the cylinder due to the water-cylinder interaction.

Assuming a harmonic solution and applying the method of the separation of variables, the transverse deflection $u$ can be expressed as 


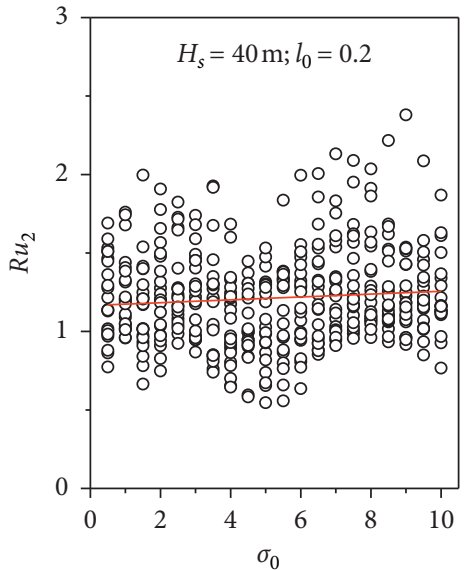

— Trend line

(a)

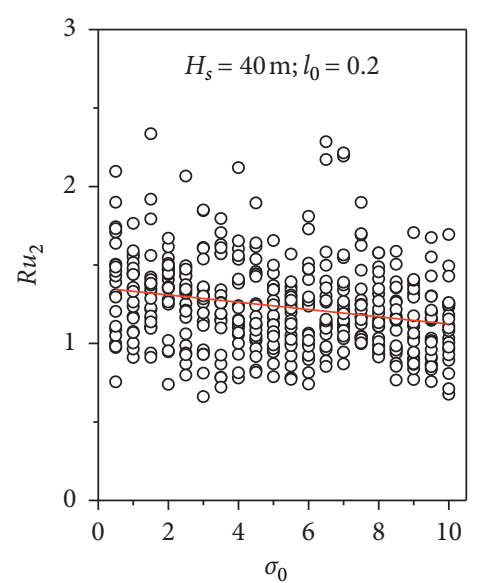

— Trend line

(b)

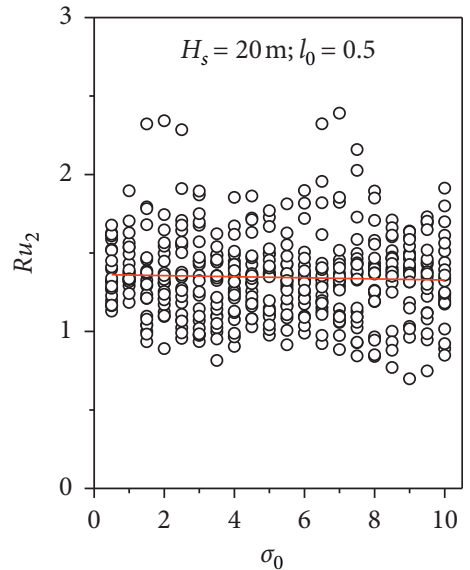

— Trend line

(c)

FIgURE 19: The parameters $R u_{2}$ versus $\sigma_{0}$ for different values of $H$ and $l_{0}$.

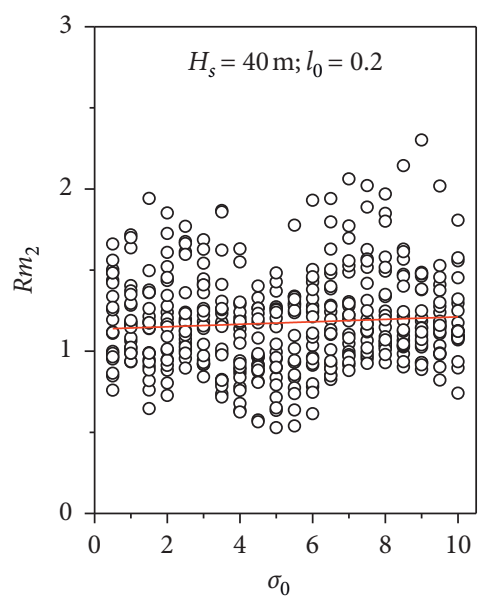

- Trend line

(a)

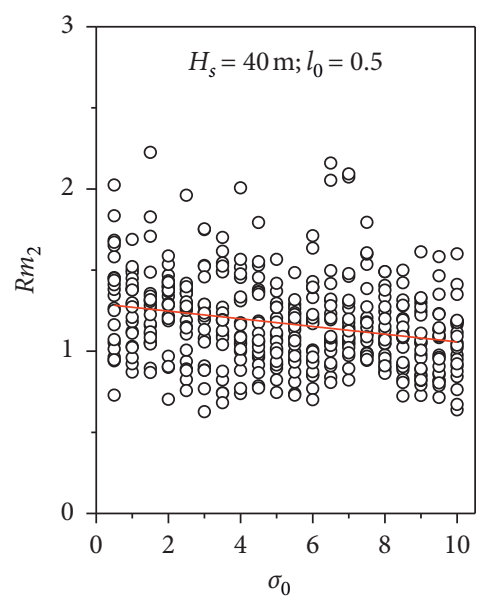

_ Trend line

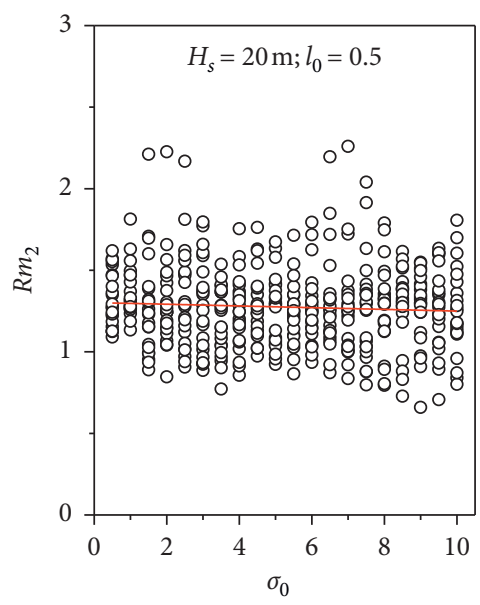

(b)

(c)

Figure 20: The parameters $R m_{2}$ versus $\sigma_{0}$ for different values of $H$ and $l_{0}$.

$$
u(z, t)=\phi(z) \mathrm{e}^{\mathrm{i} \omega t}
$$

Substituting (39) into (38), we obtain

$$
E I_{c} \frac{\partial^{4} \phi}{\partial z^{4}}-\left(\rho_{c} A_{c}+m_{w}\right) \omega^{2} \phi=0 .
$$

The four boundary conditions associated with the equations of motion in (40) can be expressed as follows:

(1) Bending moment at $z=0$ :

$$
E I_{c} \frac{\partial^{2} \phi}{\partial z^{2}}-k_{r} \frac{\partial \phi}{\partial z}=0 \mid
$$

(2) Shear force at $z=0$ :

$$
E I_{c} \frac{\partial^{3} \phi}{\partial z^{3}}+k_{h} \phi=0
$$

(3) Bending moment at $z=H$ :

$$
E I_{c} \frac{\partial^{2} \phi}{\partial z^{2}}=0
$$

(4) Shear force at $z=H$ : 


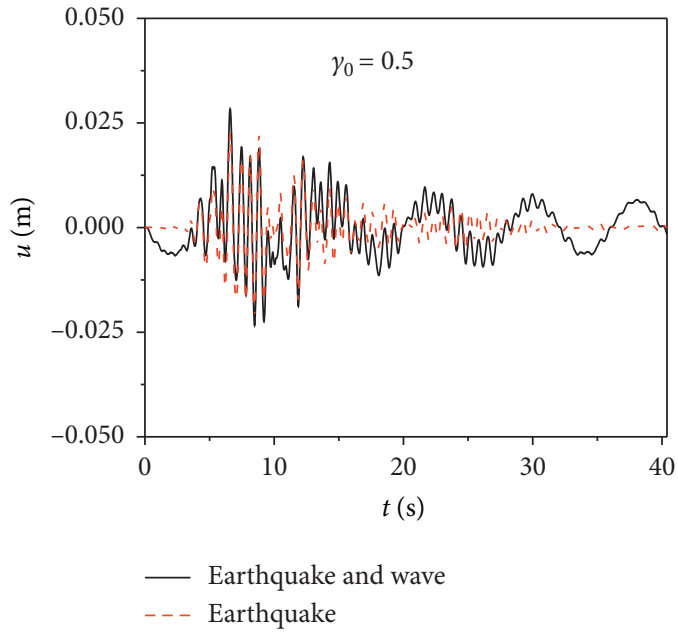

(a)

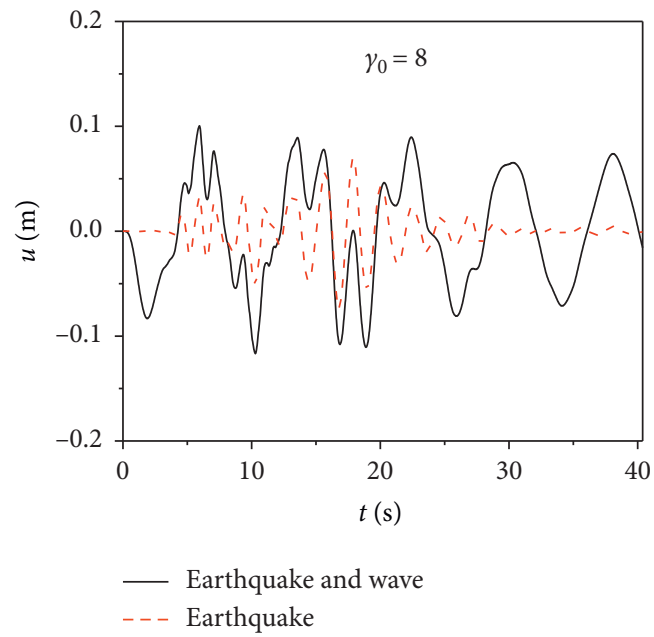

(b)

FIgURE 21: Relative displacement on top of the cylinder under the combined action of earthquakes and waves and under the HECTOR earthquake (ID number 4 in Table 2).

TABLe 2: Details of the ground motion.

\begin{tabular}{|c|c|c|c|c|c|}
\hline \multirow{2}{*}{ ID number } & \multicolumn{3}{|c|}{ PEER-NGA record information } & \multicolumn{2}{|c|}{ Recorded motions } \\
\hline & Record seq. number & Lowest freq. (Hz.) & File names-horizontal records & $P G A_{\max }(\mathrm{g})$ & $P G V_{\max }(\mathrm{cm} / \mathrm{s})$. \\
\hline 1 & 953 & 0.25 & NORTHR/MUL009 & 0.52 & 63 \\
\hline 2 & 960 & 0.13 & NORTHRLOS000 & 0.48 & 45 \\
\hline 3 & 1602 & 0.06 & DUZCE/BOL000 & 0.82 & 62 \\
\hline 4 & 1787 & 0.04 & HECTOR/HEC00 & 0.34 & 42 \\
\hline 5 & 169 & 0.06 & IMPVALUHDLT262 & 0.35 & 33 \\
\hline 6 & 174 & 0.25 & IMPVALHE11140 & 0.38 & 42 \\
\hline 7 & 1111 & 0.13 & KOBENISO00 & 0.51 & 37 \\
\hline 8 & 1116 & 0.13 & KOBE/SHI000 & 0.24 & 38 \\
\hline 9 & 1158 & 0.24 & KOCAELUDZC1B0 & 0.36 & 59 \\
\hline 10 & 1148 & 0.09 & KOCAELIARC000 & 0.22 & 40 \\
\hline 11 & 900 & 0.07 & LANDERSYER270 & 0.24 & 52 \\
\hline 12 & 848 & 0.13 & LANDERSICLW-LN & 0.42 & 42 \\
\hline 13 & 752 & 0.13 & LOMAPICAP000 & 0.53 & 35 \\
\hline 14 & 767 & 0.13 & LOMAPG03000 & 0.56 & 45 \\
\hline 15 & 1633 & 0.13 & MANJILABBAR-L & 0.51 & 54 \\
\hline 16 & 721 & 0.13 & SUPERSTIB-ICO00 & 0.36 & 46 \\
\hline 17 & 725 & 0.25 & SUPERSTIB-POE270 & 0.45 & 36 \\
\hline 18 & 829 & 0.07 & CAPEMENDRIO270 & 0.55 & 44 \\
\hline 19 & 1244 & 0.05 & CHICHICHY1Q1-E & 0.44 & 115 \\
\hline 20 & 1485 & 0.05 & CHICHITCU045-E & 0.51 & 39 \\
\hline 21 & 68 & 0.25 & SFERNPELO9O & 0.21 & 19 \\
\hline 22 & 125 & 0.13 & FRIULIA-TMZO00 & 0.35 & 31 \\
\hline
\end{tabular}

$$
E I_{c} \frac{\partial^{3} \phi}{\partial z^{3}}=-\omega^{2} M_{S} \phi
$$

Generally, the differential equations of motion in (40) do not possess an exact solution. In addition, the added mass and stiffness of the foundation change along the cylinder and pile. Therefore, the transfer matrix method (TMM) is used to solve the previous equations of motion. During analysis by the TMM, the cylinder and pile are discretized into $L$ continuous beam elements as shown in Figure 3, and the equation of motion for each element is derived. The added mass and stiffness of the foundation are assumed to be constant along each element. The analytical solution of the motion for each element is derived in the following section.

4.1. Free Vibration. The differential equation of motion for the $i$ th element can be written as

$$
E I_{c} \frac{\partial^{4} \phi_{(i)}}{\partial z^{4}}-\left(m_{c}+M_{w}\right) \omega^{2} \phi_{(i)}=0,
$$




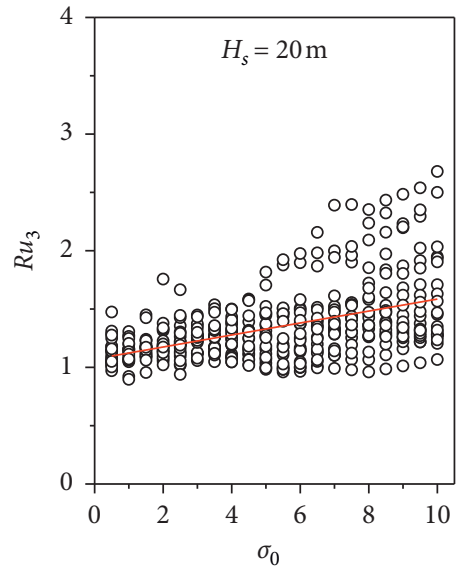

- Trend line

(a)

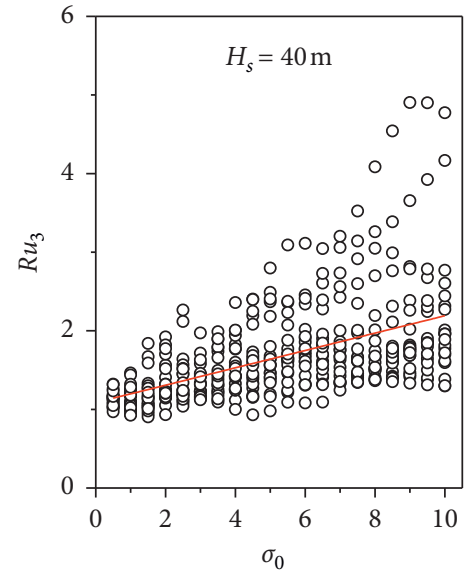

— Trend line

(b)

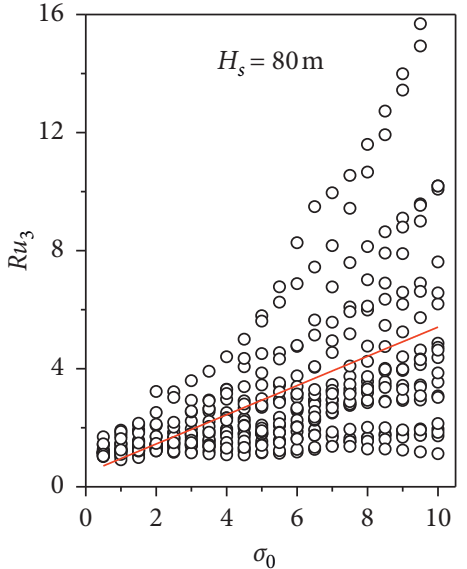

- Trend line

(c)

FIGURE 22: The parameters $R u_{3}$ versus $\sigma_{0}$ for different values of $H$ with $l_{0}=0.2$.

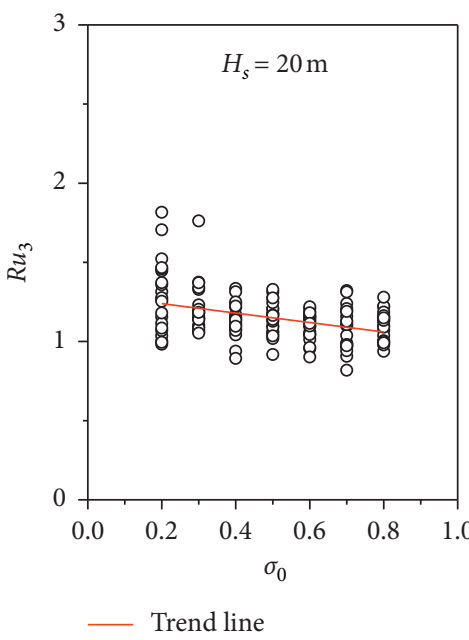

(a)

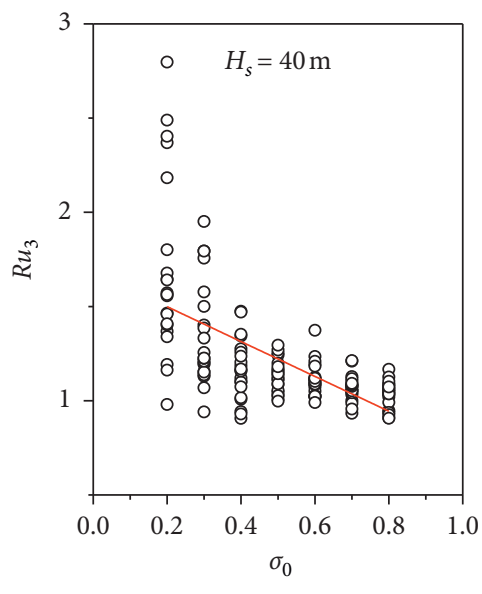

— Trend line

(b)

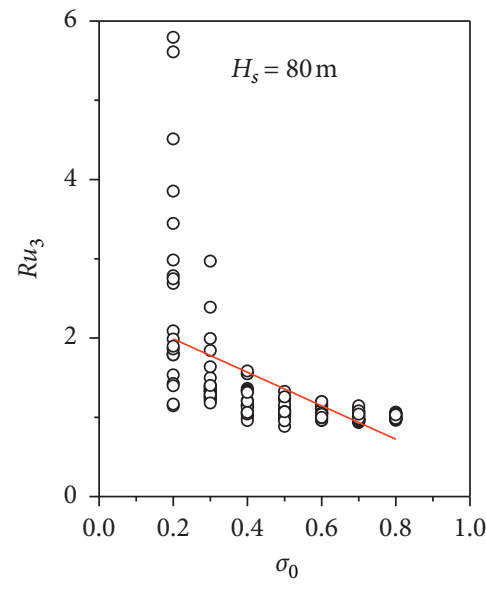

- Trend line

(c)

Figure 23: The parameters $R u_{3}$ versus $l_{0}$ for different values of $H$ with $\sigma_{0}=5$.

where $m_{c}=\rho_{c} A_{c}, M_{w}=\int_{z_{i-1}}^{z_{i}} m_{w}(z) \mathrm{d} z / l_{i}$ is the equivalent added mass, $l_{i}=\left(z_{i}-z_{i-1}\right)$, and $\omega$ is the angular frequency. The general solution form of (42) can be expressed as follows:

$$
\begin{aligned}
\phi_{(i)}(z)= & A_{i} \sin \left(\beta_{i} \bar{z}_{i}\right)+B_{i} \cos \left(\beta_{i} \bar{z}_{i}\right) \\
& +C_{i} \sin h\left(\beta_{i} \bar{z}_{i}\right)+D_{i} \cos h\left(\beta_{i} \bar{z}_{i}\right), \\
\beta_{i}= & {\left[\frac{\left(m_{c}+M_{w}\right) \omega^{2}}{E I_{c}}\right]^{0.25}, }
\end{aligned}
$$

where $\bar{z}_{i}(z)=z-z_{i-1} z_{i} \leq z \leq z_{i-1}$.

According to the continuity conditions of the displacement, slope, shear force, and bending moment between the $i$ th and $(i+1)$ th elements, we obtain

$$
A_{(i+1)}=Z_{(i)} A_{(i)},
$$

in which $Z_{(i)}$ derived in Appendix is the transfer matrix; the undetermined coefficients $A_{(i+1)}$ and $A_{(i)}$ are expressed by

$$
\begin{aligned}
A_{(i)} & =\left\{A_{i} B_{i} C_{i} D_{i}\right\}^{\mathrm{T}}, \\
A_{(i+1)} & =\left\{A_{i+1} B_{i+1} C_{i+1} D_{i+1}\right\}^{\mathrm{T}} .
\end{aligned}
$$

By using the TMM, the relationship between $Z_{(L)}$ and $Z_{(1)}$ can be given as follows:

$$
\begin{aligned}
A_{(L)} & =Z A_{(1)}, \\
Z & =Z_{(L-1)} Z_{(L-2)} \cdots Z_{(2)} Z_{(1)} .
\end{aligned}
$$




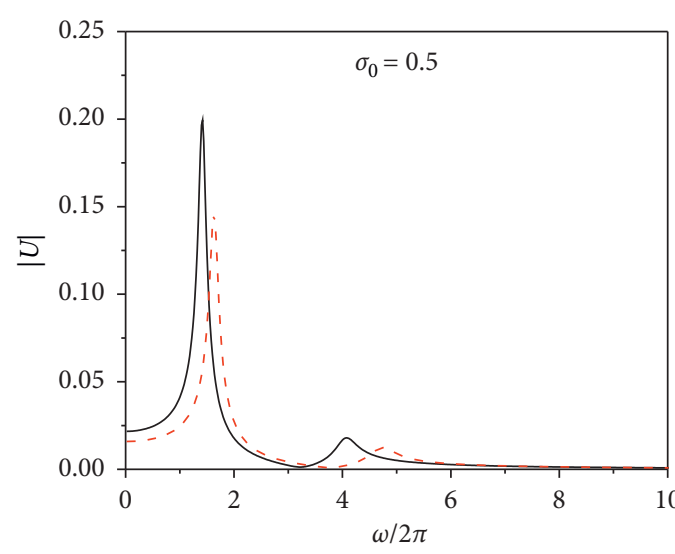

— FSSI

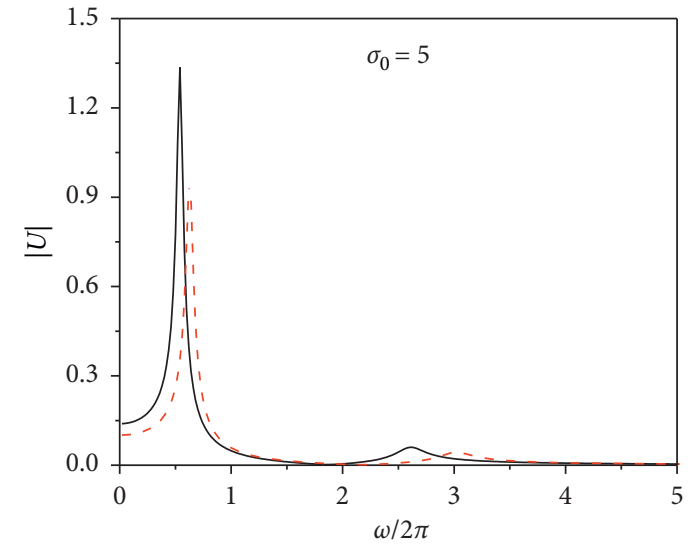

— FSSI

(a)

(b)

Figure 24: The displacement amplitude of the cylinder on the top with respect to $\omega$ with $\gamma_{0}=0.5$.

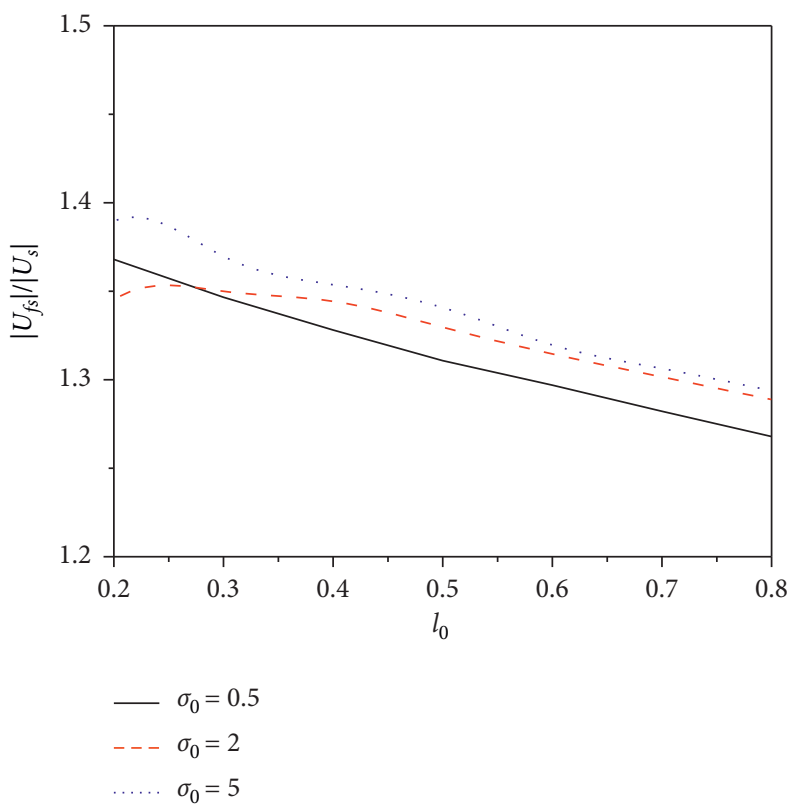

Figure 25: The variation in the ratio $\left|U_{f s}\right| /\left|U_{s}\right|$ with respect to $l_{0}$ for different values of $\sigma_{0}$ with $\gamma_{0}=0.5$.

According to the boundary conditions in (41a) and (41b), we obtain

$$
\begin{aligned}
\Gamma_{1} A_{(1)} & =0, \\
\Gamma_{1} & =\left[\begin{array}{cc}
-k_{r} & -E I_{c} \beta_{1}^{3} \\
-E I_{c} \beta_{1} & k_{h} \\
-k_{r} & E I_{c} \beta_{1}^{3} \\
E I_{c} \beta_{1} & k_{h}
\end{array}\right] .
\end{aligned}
$$

According to boundary conditions in (41c) and (41d), we obtain

$$
\Lambda A_{(L)}=0,
$$

$\Lambda=\left[\begin{array}{cc}-\sin \left(\beta_{L} l_{L}\right) & -E I_{c} \beta_{L}^{3} \cos \left(\beta_{L} l_{L}\right)+M_{S} \omega^{2} \sin \left(\beta_{L} l_{L}\right) \\ -\cos \left(\beta_{L} l_{L}\right) & E I_{c} \beta_{L}^{3} \sin \left(\beta_{L} l_{L}\right)+M_{S} \omega^{2} \cos \left(\beta_{L} l_{L}\right) \\ \sin h\left(\beta_{L} l_{L}\right) & E I_{c} \beta_{L}^{3} \cosh \left(\beta_{L} l_{L}\right)+M_{S} \omega^{2} \sin h\left(\beta_{L} l_{L}\right) \\ \cos h\left(\beta_{L} l_{L}\right) & E I_{c} \beta_{L}^{3} \sin h\left(\beta_{L} l_{L}\right)+M_{S} \omega^{2} \cos h\left(\beta_{L} l_{L}\right)\end{array}\right]^{T}$

Substituting (47) into equations ((50a), (50b)), we get

$$
\Lambda Z A_{(1)}=\Gamma_{2} A_{(1)}=0 .
$$

Furthermore, using (49a) into (51), we obtain 


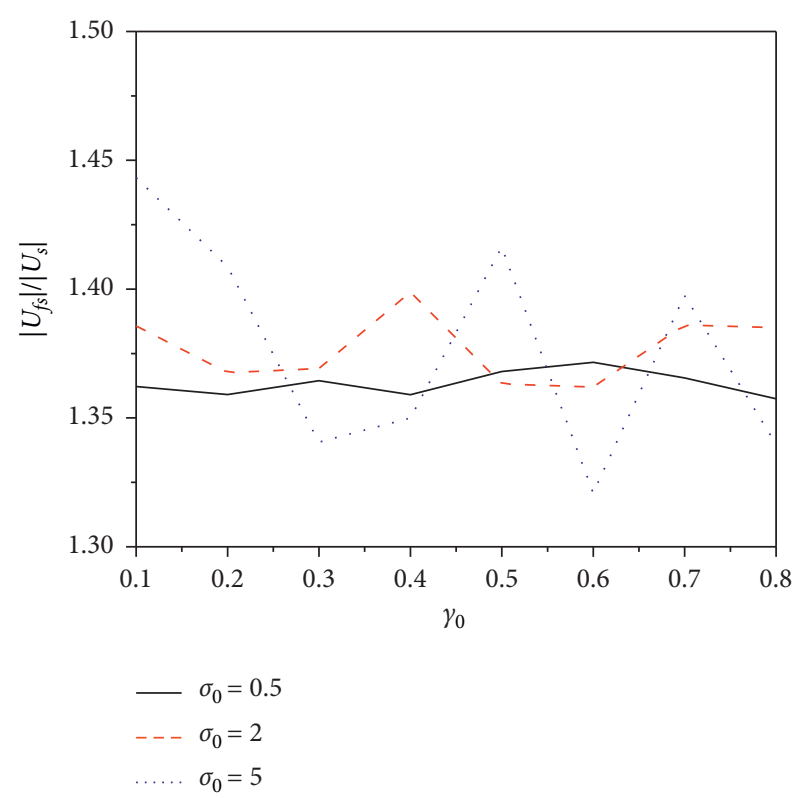

FIGURE 26: The variation in the ratio $\left|U_{f s}\right| /\left|U_{s}\right|$ with respect to $\gamma_{0}$ for different values of $\sigma_{0}$ with $l_{0}=0.2$.

$$
\begin{aligned}
\Gamma A_{(1)} & =0, \\
\Gamma & =\left[\begin{array}{l}
\Gamma_{1} \\
\Gamma_{2}
\end{array}\right] .
\end{aligned}
$$

The constant vector in (53) cannot be zero. Therefore, the equation governing the natural frequencies of the cylinder is written as

$$
\left|\begin{array}{l}
\Gamma_{1} \\
\Gamma_{2}
\end{array}\right|=0
$$

The natural frequencies of the system denoted by $\omega_{j}$ can be obtained by solving (54), and the corresponding mode shape is denoted by $\phi_{j}$. It should be noted that (54) is a transcendental equation that needs to be solved numerically. In this study, (54) is solved using MATLAB software.

4.2. Dynamic Responses in the Time Domain. By using the mode superposition method, the deformation of the cylinder can be written as follows:

$$
u=\sum_{j=1}^{\infty} \phi_{j}(z) q_{j}(t)
$$

where $q_{j}(t)$ are the generalized coordinates and the damping ratio is denoted by $\xi_{j}$. Substituting (55) into (1), considering the damping and using the orthogonality of the mode shapes, the generalized coordinates $q_{j}(t)$ in the equation of motion of the cylinder subjected to earthquake and wave actions are expressed as [5]

$$
\begin{aligned}
\left(M_{j}+M_{w j}\right) \ddot{q}_{j}+C_{j} \dot{q}_{j}+K_{j} q_{j}=-M_{0 j} \ddot{u}_{g}-M_{0 w j} \ddot{u}_{g}+F_{j}^{w} \\
M_{j}=\int_{0}^{H} m_{c} \phi_{j}(z)^{2} \mathrm{~d} z \\
M_{0 j}=\int_{0}^{H} m_{c} \phi_{j}(z) \mathrm{d} z \\
K_{j}=\omega_{j}^{2}\left(M_{j}+M_{w j}\right) \\
C_{j}=2 \xi_{j} \omega_{j}\left(M_{j}+M_{w j}\right) \\
M_{w j}=\int_{0}^{h} m_{w} \phi_{j}(z)^{2} \mathrm{~d} z \\
M_{0 w j}=\int_{0}^{h} m_{w} \phi_{j}(z) \mathrm{d} z \\
F_{j}^{w}=\int_{0}^{h} f_{w} \phi_{j}(z) \mathrm{d} z
\end{aligned}
$$

Equation (56) is a dynamic equation of a single-degreeof-freedom system. It can be solved by the standard implicit time integration algorithm such as the Newmark or Wilson method. In addition to the implicit algorithm, it can also be solved by the second-order accurate explicit algorithm proposed by the authors [31].

4.3. Dynamic Responses in the Frequency Domain. The deformation of the cylinder under earthquake action can be written as follows:

$$
U=\sum_{j=1}^{\infty} \phi_{j}(z) Q_{j}(\omega),
$$

where $Q_{j}(\omega)$ is the frequency generalized coordinates, which can be calculated by

$$
Q_{j}(\omega)=\frac{-\left(M_{0 j}+M_{0 w j}\right) A_{g}}{\left(M_{j}+M_{w j}\right)\left[\omega_{j}^{2}+2 i \xi_{j} \omega_{j} \omega-\omega^{2}\right]},
$$

where $A_{g}$ is the ground acceleration in the frequency domain.

\section{Results and Discussion}

The material parameters of the flexible cylinder, including Young's modulus, density, and damping ratio, are $30000 \mathrm{MPa}, 2500 \mathrm{~kg} / \mathrm{m}^{3}$, and 0.05 , respectively. The water depth is equal to the height of the cylinder. The peak acceleration of the ground motion $\left(a_{\max }\right)$ is equal to $0.1 \mathrm{~g}$. The wave height and wave period are $H_{w}=3 \mathrm{~m}$ and $T=8 \mathrm{~s}$, respectively. The translational and rotational spring constants $k_{h}$ and $k_{r}$ for a rigid circular massless foundation with radius $R_{0}$ on deep soil strata can be evaluated by the following frequency-independent expressions [32]: 


$$
\begin{aligned}
& k_{h}=\frac{8 G R_{0}}{2-\mu}, \\
& k_{r}=\frac{8 G R_{0}^{3}}{3(1-\mu)},
\end{aligned}
$$

where $G$ and $\mu=0.3$ are the shear modulus and Poisson's ratio of the soil, respectively.

The effects of the fluid-structure interaction (FSI), soilstructure interaction (SSI), and fluid-structure-soil interaction (FSSI) on the water-cylinder-soil system can be better understood by studying the system dynamic properties in terms of dimensionless parameters. Therefore, four dimensionless parameters, including the width-depth ratio $\left(l_{0}\right)$, mass ratio $\left(\delta_{0}\right)$, height-radius ratio $\left(\gamma_{0}\right)$, and stiffness ratio $\left(\sigma_{0}\right)$, are introduced as

$$
\begin{aligned}
l_{0} & =\frac{2 a}{h}, \\
\delta_{0} & =\frac{M_{S}}{\rho_{c} A_{c} H}, \\
\gamma_{0} & =\frac{H}{R_{0}}, \\
\sigma_{0} & =\frac{E I_{c}}{H^{3} G R_{0}} .
\end{aligned}
$$

5.1. Validation. The solutions for the earthquake-induced pressure and wave-induced pressure are first verified. Figure 4 shows the comparison of the hydrodynamic pressure $P_{j}$ on a circular cylinder between the present SBFEM and the finite element method (FEM) developed by Wang et al. [33]. Figure 5 shows the comparison of the total wave forces $F_{w}$ on a circular cylinder computed by the present SBFEM and FEM developed by Wang et al. [33], where ${ }_{F}^{w}=\int_{0}^{h} f_{w}(z) \mathrm{d} z$. It can be seen that the proposed SBFEM agrees well with the FEM.

The solution of the free vibration is further validated by assuming the foundation rigid. The first natural frequencies of the circular cylinders vibrating in water are compared with those in a previous study [15], where $E=29.4 \mathrm{GPa}$, $\rho_{c}=2450 \mathrm{~kg} / \mathrm{m}^{3}$, and $H=h=20 \mathrm{~m}$. The natural frequencies and the difference between the two studies are tabulated in Table 1. It is evident that good agreement is observed.

The solution of the dynamic response is finally verified by assuming the foundation rigid. Figure 6 shows the comparison of the displacement amplitude at the top of a circular cylinder excited by the harmonic ground motion between the proposed solution and the experimental values [34]. It can be seen that good agreement is observed. Figure 7 shows the comparison of the displacement time history at the top of a circular cylinder excited by wave forces between the proposed solution and the software ABAQUS, where $E=29.4 \mathrm{GPa}, \quad \rho_{c}=2450 \mathrm{~kg} / \mathrm{m}^{3}, \quad a=2 \mathrm{~m}, \quad H=h=20 \mathrm{~m}$, $H_{w}=2 \mathrm{~m}$, and $\omega_{0}=0.1 \pi$. It can also be seen that good agreement is observed.
5.2. Free Vibration. The fundamental frequencies of the cylinder in air with a rigid foundation, considering the SSI, FSI, and FSSI, are denoted by $\omega_{0}, \omega_{s}, \omega_{f}$, and $\omega_{f s}$.

The effects of the SSI on the fundamental frequency of the cylinder are first investigated. Figure 8 shows the ratio $\omega_{s} / \omega_{0}$ with respect to $\sigma_{0}$ for different values of $l_{0}$ and $H$ with $\delta_{0}=0$ and $\gamma_{0}=0.5$. It can be seen that the ratio $\omega_{s} / \omega_{0}$ decreases as $\sigma_{0}$ increases, which means that the fundamental frequency of the cylinder decreases when the stiffness of the foundation decreases. It can also be seen that the ratio $\omega_{s} / \omega_{0}$ is independent of $l_{0}$ and $H$. Figure 9 shows the ratio $\omega_{s} / \omega_{0}$ with respect to $\sigma_{0}$ for different values of $\delta_{0}$ with $\gamma_{0}=0.5$. It can be seen that the ratio $\omega_{s} / \omega_{0}$ increases as $\delta_{0}$ increases which means that the effects of the SSI on the fundamental frequency of the cylinder decrease as $\delta_{0}$ increases. Figure 10 shows the ratio $\omega_{s} / \omega_{0}$ with respect to $\gamma_{0}$ for different values of $\sigma_{0}$ with $\delta_{0}=0$. It can be seen that the ratio $\omega_{s} / \omega_{0}$ decreases marginally with increasing $\gamma_{0}$.

The effects of the FSI on the fundamental frequency of the cylinder are investigated next. Figure 11 shows the ratio $\omega_{s} / \omega_{0}$ with respect to $l_{0}$ for different values of $\delta_{0}$ and $H$. It can be seen that the ratio $\omega_{s} / \omega_{0}$ increases as $l_{0}$ and $\delta_{0}$ increase, which means that the effects of the FSI on the fundamental frequency of the cylinder decrease as $l_{0}$ and $\delta_{0}$ increase. It can also be seen that the ratio $\omega_{s} / \omega_{0}$ is independent of $H$. Figure 12 shows the ratio $\omega_{f s} / \omega_{s}$ with respect to $\sigma_{0}$ for different values of $\gamma_{0}$ with $\delta_{0}=0$ and $l_{0}=0.2$. It can be seen that the ratio $\omega_{f s} / \omega_{s}$ decreases marginally with the increase in $\sigma_{0}$ and decrease in $\gamma_{0}$.

The effects of the SSI and FSI on the vibration shapes of the cylinder are thirdly investigated, where $H=8 \mathrm{~m}, l_{0}=0.1$, $\delta_{0}=0$, and $\gamma_{0}=0.5$. Figure 13 shows the vibration mode shapes of the cylinder in a vacuum for different values of $G$ with $H=8 \mathrm{~m}, l_{0}=0.1, \delta_{0}=0$, and $\gamma_{0}=0.5$. The SSI has a substantial effect on the vibration mode shapes of the cylinder when the soil stiffness is weak, especially for the highorder mode. Figure 14 shows the vibration mode shapes of the cylinder in a vacuum and in water with $H=8 \mathrm{~m}$, $G=50 \mathrm{MPa}, l_{0}=0.1, \delta_{0}=0$, and $\gamma_{0}=0.5$. It can be seen that the FSI has little effect on the fundamental vibration modes, while it has a slight effect on the higher modes.

5.3. Dynamic Responses in the Time Domain. The dominant frequency of the seismic waves is substantially different for the different project sites. The far-field suite of 22 records introduced in the FEMA-P695 [35] selected from the NGAWest 2 ground motion database of the Pacific Earthquake Engineering Research Center is also considered in the present study. The details of the three ground motions are given in Table 1. The dynamic responses of the pier are investigated when the peak acceleration $\left(a_{\max }\right)$ is equal to $0.1 \mathrm{~g}, \delta_{0}=0, H_{w}=3 \mathrm{~m}$, and the wave period $T_{w}=8 \mathrm{~s}$.

The effects of the SSI on the seismic responses of the cylinder are first investigated. The peak displacements on the top of the cylinder with rigid foundation and considering the SSI are denoted by $u_{0 \max }$ and $u_{1 \max }$, respectively. The peak bending moment on the base of the cylinder with a rigid foundation and considering the SSI are denoted by $b m_{0 \max }$ 
and $b m_{1 \mathrm{max}}$, respectively. Two dimensionless parameters are introduced as $R u_{1}=u_{1 \max } / u_{0 \max }$ and $R m_{1}=b m_{1 \max } / b m_{0 \max }$. Figures 15 and 16 show the parameters $R u_{1}$ and $R m_{1}$ versus $\sigma_{0}$ for different values of $H$ with $l_{0}=0.2$ and $\gamma_{0}=0.5$. Figures 17 and 18 show the parameters $R u_{1}$ and $R m_{1}$ versus $l_{0}$ for different values of $H$ with $\sigma_{0}=5$ and $\gamma_{0}=0.5$. The SSI increases the displacement of the cylinder and this influence increases as $\sigma_{0}$ and $l_{0}$ increase and $H$ decreases, which means that the SSI has greater effect on the dynamic response of the squatty cylinder. The bending moment of the cylinder may decrease due to the SSI when $H$ is large and $l_{0}$ is small, while it may increase due to the SSI when $H$ is small and $l_{0}$ is large.

The effects of the FSI on the seismic responses of the cylinder are secondly investigated next, where $\gamma_{0}=0.5$. The peak displacement on the top of the cylinder and the peak bending moment on the base of the cylinder considering the FSSI are denoted by $u_{2 \max }$ and $b m_{2 \max }$, respectively. Two dimensionless parameters are introduced as $R u_{2}=u_{2 \max } /$ $u_{1 \max }$ and $R m_{2}=b m_{2 \max } / b m_{1 \text { max }}$. Figures 19 and 20 show the parameters $R u_{2}$ and $R m_{2}$ versus $\sigma_{0}$ for different values of $H$ and $l_{0}$. It can be seen that the FSI increases the displacement and bending moment of the cylinder. However, the parameters $R u_{2}$ and $R m_{2}$ have no obvious tendency as $\sigma_{0}$ increases.

The effects of wave forces on the dynamic responses of the cylinder are investigated thirdly, where $\gamma_{0}=0.5$. The peak displacements on the top of the cylinder under the combined action of earthquakes and waves are denoted by $u_{3 \max }$. A dimensionless parameter is introduced as $R u_{3}=u_{3 \max } / u_{2 \max }$. Figure 21 shows the history of the relative displacement on top of the cylinder under the combined action of earthquakes and waves under the HECTOR earthquake (ID number 4 in Table 2), where $l_{0}=0.2, \gamma_{0}=0.5$, and $H=40 \mathrm{~m}$. Figure 22 shows the parameters $R u_{3}$ versus $\sigma_{0}$ for different values of $H$ with $l_{0}=0.2$. Figure 23 shows the parameters $R u_{3}$ versus $l_{0}$ for different values of $H$ with $\sigma_{0}=5$. The wave forces substantially increase the dynamic responses of the cylinder and this influence increases as $\sigma_{0}$ increases and $l_{0}$ decreases. It can also be seen from Figure 17 that the dynamic responses of the cylinder under the combined action of earthquakes and waves display obvious periodicity.

5.4. Dynamic Responses in the Frequency Domain. The effects of the FSI on the frequency responses of the cylinder are investigated in this section, where $H=40 \mathrm{~m}$ and $\delta_{0}=0$.

Figure 24 shows the displacement amplitude of the cylinder on the top with respect to the earthquake frequency in the case of the SSI and FSSI, where $l_{0}=0.2$ and $\gamma_{0}=0.5$. The frequency responses of the cylinder increase due to the FSI. Figure 25 shows the ratio $\left|U_{f s}\right| /\left|U_{s}\right|$ with respect to $l_{0}$ for different values of $\sigma_{0}$ with $\gamma_{0}=0.5$. Figure 26 shows the ratio $\left|U_{f_{s}}\right| /\left|U_{s}\right|$ with respect to $\gamma_{0}$ for different values of $\sigma_{0}$ with $l_{0}=0.2$. It can be seen that the ratio $\left|U_{f s}\right| /\left|U_{s}\right|$ decreases marginally as $l_{0}$ increases. In general, the dynamic responses of the cylinder increase due to the FSI by approximately $30 \% \sim 40 \%$.

\section{Conclusions}

The effects of fluid-structure-soil interaction on the dynamic response of offshore cylinders are investigated by idealizing the cylinder as a uniform beam, replacing the water-cylinder interaction with added mass, and replacing the soil-structure interaction with translational and rotational springs. The earthquake-induced and wave-induced pressure on a circular cylinder are determined by the scaled boundary finite element method. The closed-form expression for the natural frequencies and mode shapes of the system is derived by using the transfer matrix method and the dynamic response of the system under the combined action of earthquakes and waves further obtained and derived by the time integration algorithm. From all the results presented in the present study, the following conclusion can be made:

(1) The SSI decreases the fundamental frequency of the cylinder and the influence increases as the stiffness ratio and height-radius ratio increase and mass ratio decreases. The FSI decreases the fundamental frequency of the cylinder and the influence increases as the width-depth ratio and mass ratio decrease. The SSI has a substantial effect on the vibration mode shapes of the cylinder when the soil stiffness is weak and the FSI has a slight effect on the higher modes.

(2) The SSI increases the displacement of the cylinder and the influence increases as the stiffness ratio and width-depth ratio increase, and the cylinder height decreases. The SSI can decrease the bending moment of the cylinder for slender cylinders with large heights, while it can increase the bending moment of the cylinder for squatty cylinders with small heights.

(3) The FSI increases the displacement and bending moment of the cylinder and the influence decreases marginally with increasing width-depth ratio. It should be noted that the effects of the FSI on the dynamic responses of the cylinder have no obvious tendency as the stiffness ratio increases.

(4) The wave forces can substantially increase the dynamic responses of the cylinder, and the influence increases as the stiffness ratio increases and the width-depth ratio decreases. The wave forces may be the dominant loading in the seismic design of offshore structures for soft soil. Consequently, it is necessary to consider the combined action of earthquakes and waves in the dynamic analysis of offshore structures.

Generally, during an earthquake, the wave condition and the soil behaviour will be significantly affected by the ground movement of the earthquake. However, the present model is a simplified model without considering the interaction between the earthquake, wave, and soil. The linear regular wave is only used to investigate the response trends of the circular cylinder subjected to earthquake and wave loadings in the present study. However, a wave subjected to an earthquake cannot be modeled as a linear regular wave in a real scenario. In practical engineering applications, the wave 
spectrum, such as the JONSWAP spectrum [36] and Pierson-Moskowitz spectrum [37], can be used to define a stationary random sea state caused by wind-generated surface waves. It should be noted that the linear wave model is not sufficient to represent the wave condition during an earthquake, which is usually highly nonlinear. Therefore, the range of applications of the present linear wave model is $h$ / $L_{0}>0.2$ and $H_{w} / / h \leq 0.2$ [38]. In future work, we will improve the present model by considering the nonlinear wave force on the cylinder.

\section{Appendix}

According to the continuity conditions of the displacement, slope, shear force, and bending moment between the $i$ th and $(i+1)$ th elements, we obtain

$$
T R_{(i+1)} A_{(i+1)}=T R_{(i)} A_{(i)}
$$

where the matrices $T R_{(i)}$ and $T R_{(i+1)}$ are expressed as

$$
\begin{aligned}
T R_{(i)} & =\left[\begin{array}{cccc}
\sin \left(\beta_{i} l_{i}\right) & \cos \left(\beta_{i} l_{i}\right) & \sin h\left(\beta_{i} l_{i}\right) & \cosh \left(\beta_{i} l_{i}\right) \\
\beta_{i} \cos \left(\beta_{i} l_{i}\right) & -\beta_{i} \sin \left(\beta_{i} l_{i}\right) & \beta_{i} \cos h\left(\beta_{i} l_{i}\right) & \beta_{i} \sin h\left(\beta_{i} l_{i}\right) \\
-E I_{c} \beta_{i}^{2} \sin \left(\beta_{i} l_{i}\right) & -E I_{c} \beta_{i}^{2} \cos \left(\beta_{i} l_{i}\right) & E I_{c} \beta_{i}^{2} \sin h\left(\beta_{i} l_{i}\right) & E I_{c} \beta_{i}^{2} \cos h\left(\beta_{i} l_{i}\right) \\
-E I_{c} \beta_{i}^{3} \cos \left(\beta_{i} l_{i}\right) & E I_{c} \beta_{i}^{3} \sin \left(\beta_{i} l_{i}\right) & E I_{c} \beta_{i}^{3} \cosh \left(\beta_{i} l_{i}\right) & E I_{e q} \beta_{i}^{3} \sin h\left(\beta_{i} l_{i}\right)
\end{array}\right], \\
T R_{(i+1)} & =\left[\begin{array}{cccc}
0 & 1 & 0 & 1 \\
\beta_{i+1} & 0 & \beta_{i+1} & 0 \\
0 & -E I_{c} \beta_{i+1}^{2} & 0 & E I_{c} \beta_{i+1}^{2} \\
-E I_{c} \beta_{i+1}^{3} & 0 & E I_{c} \beta_{i+1}^{3} & 0
\end{array}\right] .
\end{aligned}
$$

Equation (A.1) can be further rewritten as

$$
A_{(i+1)}=T R_{(i+1)}^{-1} T R_{(i)} A_{(i)}=Z_{(i)} A_{(i)},
$$

where $Z_{(i)}=T R_{(i+1)}{ }^{-1} T R_{(i)}$.

\section{Data Availability}

No data were used to support this study.

\section{Conflicts of Interest}

The authors declare that there are no conflicts of interest regarding the publication of this paper.

\section{Acknowledgments}

This work was supported by the National Natural Science Foundation of China (51678015 and 51708010) and Ministry of Education Innovation Team of China (IRT_17R03).

\section{References}

[1] X. Y. Zheng, H. Li, W. Rong, and W. Li, "Joint earthquake and wave action on the monopile wind turbine foundation: an experimental study," Marine Structures, vol. 44, pp. 125-141, 2015.

[2] R. P. S. Han and H. Xu, "A simple and accurate added mass model for hydrodynamic fluid-structure interaction analysis," Journal of the Franklin Institute, vol. 333, no. 6, pp. 929-945, 1996.

[3] B. Song, F. Zheng, and Y. Li, "Study on a simplified calculation method for hydrodynamic pressure to slender structures under earthquakes," Journal of Earthquake Engineering, vol. 17, no. 5, pp. 720-735, 2013.
[4] K. Wei, W. Yuan, and N. Bouaanani, "Experimental and numerical assessment of the three-dimensional modal dynamic response of bridge pile foundations submerged in water," Journal of Bridge Engineering, vol. 18, no. 10, pp. 1032-1041, 2013.

[5] C.-Y. Liaw and A. K. Chopra, "Dynamics of towers surrounded by water," Earthquake Engineering \& Structural Dynamics, vol. 3, no. 1, pp. 33-49, 1974.

[6] A. N. Williams, "Earthquake response of submerged circular cylinder," Ocean Engineering, vol. 13, no. 6, pp. 569-585, 1986.

[7] Y. Tanaka and R. T. Hudspeth, "Restoring forces on vertical circular cylinders forced by earthquakes," Earthquake Engineering \& Structural Dynamics, vol. 16, no. 1, pp. 99-119, 1988.

[8] X. Du, P. Wang, and M. Zhao, "Simplified formula of hydrodynamic pressure on circular bridge piers in the time domain," Ocean Engineering, vol. 85, pp. 44-53, 2014.

[9] P. Wang, M. Zhao, H. Li, and X. Du, "An accurate and efficient time-domain model for simulating water-cylinder dynamic interaction during earthquakes," Engineering Structures, vol. 166, pp. 263-273, 2018.

[10] A. Goyal and A. K. Chopra, "Simplified evaluation of added hydrodynamic mass for intake towers," Journal of Engineering Mechanics, vol. 115, no. 7, pp. 1393-1412, 1989.

[11] Q. Li and W. Yang, "An improved method of hydrodynamic pressure calculation for circular hollow piers in deep water under earthquake," Ocean Engineering, vol. 72, pp. 241-256, 2013.

[12] W. Yang and Q. Li, "A new added mass method for fluidstructure interaction analysis of deep-water bridge," KSCE Journal of Civil Engineering, vol. 17, no. 6, pp. 1413-1424, 2013.

[13] H. Jiang, B. Wang, X. Bai, C. Zeng, and H. Zhang, "Simplified expression of hydrodynamic pressure on deep water cylindrical bridge piers during earthquakes," Journal of Bridge Engineering, vol. 22, no. 6, Article ID 04017014, 2017. 
[14] P. Wang, M. Zhao, and X. Du, "Analytical solution and simplified formula for earthquake induced hydrodynamic pressure on elliptical hollow cylinders in water," Ocean Engineering, vol. 148, pp. 149-160, 2018.

[15] P. Wang, M. Zhao, and X. Du, "A simple added mass model for simulating elliptical cylinder vibrating in water under earthquake action," Ocean Engineering, vol. 179, pp. 351-360, 2019.

[16] P. Wang, M. Zhao, and X. Du, "Simplified formula for earthquake-induced hydrodynamic pressure on round-ended and rectangular cylinders surrounded by water," Journal of Engineering Mechanics-ASCE, vol. 145, no. 2, Article ID 04018137, 2019b.

[17] J. R. Morison, J. W. Johnson, S. A. Schaaf, and S. A. Schaaf, "The force exerted by surface waves on piles," Journal of Petroleum Technology, vol. 2, no. 5, pp. 149-154, 1950.

[18] R. C. MacCamy and R. A. Fuchs, Wave forces on piles: a diffraction theory. Beach Erosion Board Technical Memorandum U.S. Army Corps of Engineers, Report 69, United States Army Corps of Engineers, Washington, DC, USA, 1954.

[19] H. S. Chen and C. C. Mei, "Wave forces on a stationary platform of elliptical shape," Journal of Ship Research, vol. 17, no. 2, pp. 61-71, 1973.

[20] A. N. Williams, "Wave forces on an elliptic cylinder," Journal of Waterway, Port, Coastal, and Ocean Engineering, vol. 111, no. 2, pp. 433-449, 1985.

[21] J. Liu, A. Guo, and H. Li, "Analytical solution for the linear wave diffraction by a uniform vertical cylinder with an arbitrary smooth cross-section," Ocean Engineering, vol. 126, pp. 163-175, 2016.

[22] B. Li, L. Cheng, A. J. Deeks, and M. Zhao, "A semi-analytical solution method for two-dimensional Helmholtz equation," Applied Ocean Research, vol. 28, no. 3, pp. 193-207, 2006.

[23] J. Penzien, M. K. Kaul, and B. Berge, "Stochastic response of offshore towers to random sea waves and strong motion earthquakes," Computers and Structures, vol. 2, no. 5-6, pp. 733-756, 1972.

[24] C. Liu, S. Zhang, and E. Hao, "Joint earthquake, wave and current action on the pile group cable-stayed bridge tower foundation: an experimental study," Applied Ocean Research, vol. 63, pp. 157-169, 2017.

[25] Y. Ding, R. Ma, Y.-D. Shi, and Z.-X. Li, "Underwater shaking table tests on bridge pier under combined earthquake and wave-current action," Marine Structures, vol. 58, pp. 301-320, 2018.

[26] B.-F. Chen, "Dynamic responses of coastal structures during earthquakes including sediment-sea-structure interaction," Soil Dynamics and Earthquake Engineering, vol. 20, no. 5-8, pp. 445-467, 2000.

[27] A. Goyal and A. K. Chopra, "Hydrodynamic and foundation interaction effects in dynamics of intake towers: earthquake responses," Journal of Structural Engineering, vol. 115, no. 6, pp. 1386-1395, 1989.

[28] C. Xu and C. C. Spyrakos, "Seismic analysis of towers including foundation uplift," Engineering Structures, vol. 18, no. 4, pp. 271-278, 1996.

[29] Y. Yamada, H. Iemura, K. Kawano, and K. Venkataramana, "Seismic response of offshore structures in random seas," Earthquake Engineering \& Structural Dynamics, vol. 18, no. 7, pp. 965-981, 1989.

[30] J. P. Wolf, The Scaled Boundary Finite Element Method, John Wiley \& Sons, Chichester, UK, 2003.

[31] M. Zhao, H. Li, S. Cao, and X. Du, "An explicit time integration algorithm for linear and non-linear finite element analyses of dynamic and wave problems," Engineering Computations, vol. 36, no. 1, pp. 161-177, 2019.

[32] G. Gazetas, "Analysis of machine foundations: state of the art," Soil Dynamic and Earthquake Engineering, vol. 2, pp. 1-42, 1983.

[33] P. Wang, M. Zhao, X. Du, and X. Cheng, "A finite element solution of earthquake-induced hydrodynamic forces and wave forces on multiple circular cylinders," Ocean Engineering, vol. 189, Article ID 106336, 2019.

[34] H. Goto and K. Toki, "Vibrational characteristics and a seismic design of submerged bridge piers," Proceeding of $3 \mathrm{rd}$ World Conference in Earthquake Engineering, pp. 107-122, 1965.

[35] ATC/FEMA, "Applied technology council/federal emergency management agancy (ATC/FEMA)," in FEMA P695: Quantification of Building Seismic Performance Factors, ATC/ FEMA, Washington, DC, USA, 2009.

[36] K. Hasselmann, T. Barnett, E. Bouws, H. Carlson, D. Cartwright, and K. Enke, Measurements of Wind-Wave Growth and Swell Decay during the Joint North Sea Wave Project (JONSWAP), Deutches Hydrographisches Institut, Hamburg, Germany, 1973.

[37] K.-Y. Oh, J.-Y. Kim, and J.-S. Lee, "Preliminary evaluation of monopile foundation dimensions for an offshore wind turbine by analyzing hydrodynamic load in the frequency domain," Renewable Energy, vol. 54, pp. 211-218, 2013.

[38] Y. Zhu, "Analyses of the range of validity for several wave theories," Hai An Gong Cheng, vol. 2, no. 2, pp. 11-27, 1983, in Chinese. 